\title{
Design and Synthesis of a MOD 13 Binary Down Counter
}

This paper was downloaded from TechRxiv (https://www.techrxiv.org).

\section{LICENSE}

CC BY 4.0

SUBMISSION DATE / POSTED DATE

$14-10-2021 / 19-10-2021$

CITATION

Assim, Ara Abdulsatar Assim (2021): Design and Synthesis of a MOD 13 Binary Down Counter. TechRxiv. Preprint. https://doi.org/10.36227/techrxiv.16810198.v1

DOI

10.36227/techrxiv.16810198.v1 


\title{
Design and Synthesis of a MOD 13 Binary Down Counter
}

\author{
Ara Abdulsatar Assim ${ }^{1,2}$ \\ ${ }^{1}$ Peter the Great St. Petersburg Polytechnic University, St. Petersburg 195251, Russia \\ ${ }^{2}$ University of Salahaddin, Erbil 44001, Iraq \\ E-mail: araabdulsattar@gmail.com \\ ORCID: https://orcid.org/0000-0002-6055-9223
}

\section{Abstract}

This work explains the process of designing and synthesizing a MOD 13 binary down counter using $180 \mathrm{~nm}$ CMOS technology transistors. The beginning of the count is a combination of $1110_{2}$, the end of the count is $0010_{2}$. Simulations are made at the circuit level (transient analysis) to verify that the circuit functions correctly, then the integrated circuit layout is prepared by connecting the components manually. Finally, the layout is simulated to see how the existence of parasitic resistances and capacitances affect the output signals and it is used to estimate the maximum allowable clock frequency $\left(f_{\text {clk }}\right)$.

Index Terms-Digital counters, Binary counters, Flip-flops, Electronic counter, MOD 13 counter.

\section{Introduction to digital counters}

\subsection{General overview}

A digital counter is an electronic device that counts how many times a specific event occurred [1-8]. Digital counters are usually driven by a clock. The most widely used scheme is a sequential circuit consisting of flip flops and a clock signal, counters can be implemented using different types of flip flops. The outputs represent the bits for example, for a MOD-16 counter, we need 4 flip flops in a cascade, they either count up or down. Counters are widely used in different industries for different applications. 


\subsection{Types of digital counters}

Digital counters have different kinds, each is suitable for a certain application, they can be broadly classified into two groups: synchronous counters and asynchronous (ripple) counters.

In synchronous counters, as the name indicates the flip flops share the same clock, meaning the output of each flip flop is connected to the input of the next element, an example is shown below:

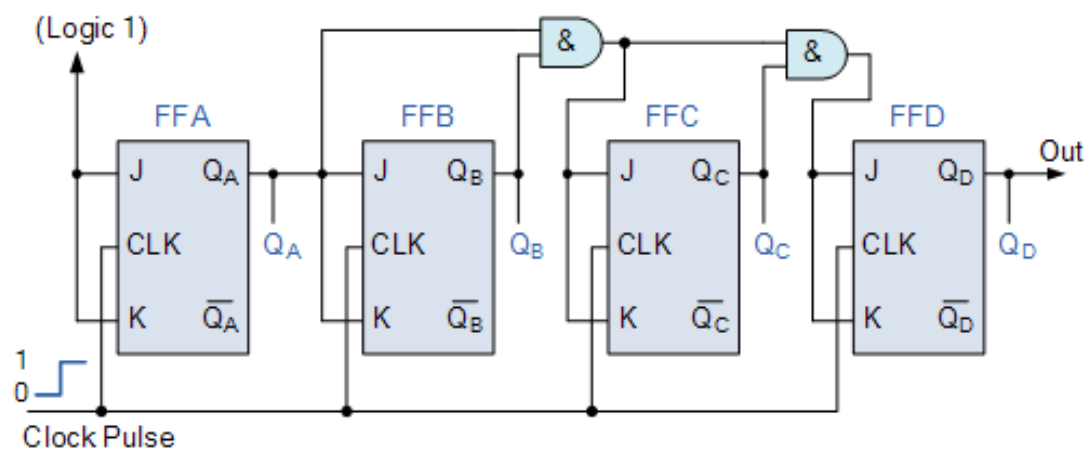

Fig 1.1 (4-bit synchronous up counter)

In contrast, asynchronous (ripple) counters work without a synchronous clock, the output of the previous flip-flop is given to the input of the following flip-flop as a clock signal which are connected in series, and the clock pulse laps through the counter. Because of the ripple clock pulse, it's also known as "ripple counter". Asynchronous (ripple) counters are able to generate $2^{\mathrm{n}}-1$ unique binary combinations.

The two types described above are very broad categories, they can be manipulated to design tens of other counters, other classified groups include: Modulus counters, up/down counters, ring counters, Johnson counters, decade counters, binary coded decimal counters, gray-code counters.

\subsection{Applications}

Counters are generally used in counting applications. They measure the time interval between two instants of time or frequency of a certain signal. Many common 
devices like processors, calculators, clocks, ovens use some kind of digital counter. They are also used in alarms, air conditioners, car parking controllers and so on.

In addition to that, they can be used as a clock divider circuit. Modern counters usually have different features, for example presetting the counter for determining the initial step. In certain cases, they are used in machinery controls. In electronics world, counters got wide usage in multiplexer circuits, digital clocks, staircase voltage generators and analog/digital converters.

\section{Design of a MOD-13 counter}

\subsection{The truth table}

The truth table can be obtained from the counter requirement (starting at 14 and ending at 2), as shown below:

\begin{tabular}{|c|c|c|c|c|c|c|c|c|}
\hline Decimal & Q3 & Q2 & Q1 & Q0 & D3 & D2 & D1 & D0 \\
\hline $\mathbf{1 5}$ & 1 & 1 & 1 & 1 & - & - & - & - \\
\hline $\mathbf{1 4}$ & 1 & 1 & 1 & 0 & 1 & 1 & 0 & 1 \\
\hline $\mathbf{1 3}$ & 1 & 1 & 0 & 1 & 1 & 1 & 0 & 0 \\
\hline $\mathbf{1 2}$ & 1 & 1 & 0 & 0 & 1 & 0 & 1 & 1 \\
\hline $\mathbf{1 1}$ & 1 & 0 & 1 & 1 & 1 & 0 & 1 & 0 \\
\hline $\mathbf{1 0}$ & 1 & 0 & 1 & 0 & 1 & 0 & 0 & 1 \\
\hline $\mathbf{9}$ & 1 & 0 & 0 & 1 & 1 & 0 & 0 & 0 \\
\hline $\mathbf{8}$ & 1 & 0 & 0 & 0 & 0 & 1 & 1 & 1 \\
\hline $\mathbf{7}$ & 0 & 1 & 1 & 1 & 0 & 1 & 1 & 0 \\
\hline $\mathbf{6}$ & 0 & 1 & 1 & 0 & 0 & 1 & 0 & 1 \\
\hline $\mathbf{5}$ & 0 & 1 & 0 & 1 & 0 & 1 & 0 & 0 \\
\hline $\mathbf{4}$ & 0 & 1 & 0 & 0 & 0 & 0 & 1 & 1 \\
\hline $\mathbf{3}$ & 0 & 0 & 1 & 1 & 0 & 0 & 1 & 0 \\
\hline $\mathbf{2}$ & 0 & 0 & 1 & 0 & 1 & 1 & 1 & 0 \\
\hline $\mathbf{1}$ & 0 & 0 & 0 & 1 & - & - & - & - \\
\hline $\mathbf{0}$ & 0 & 0 & 0 & 0 & - & - & - & - \\
\hline
\end{tabular}




\subsection{Karnaugh-maps and output expressions}

The flip-flop output expressions can be obtained using Karnaugh-maps, as following:

\begin{tabular}{|c|c|c|c|c|c|}
\hline D3 & \multicolumn{5}{|c|}{ Q1 Q0 } \\
\hline \multirow{5}{*}{ Q3Q2 } & & $\mathbf{0 0}$ & $\mathbf{0 1}$ & $\mathbf{1 1}$ & $\mathbf{1 0}$ \\
\cline { 2 - 6 } & $\mathbf{0 0}$ & $\mathrm{X}$ & $\mathrm{X}$ & 0 & 1 \\
\cline { 2 - 6 } & $\mathbf{0 1}$ & 0 & 0 & 0 & 0 \\
\cline { 2 - 6 } & 11 & 1 & 1 & $\mathrm{X}$ & 1 \\
\cline { 2 - 6 } & $\mathbf{1 0}$ & 0 & 1 & 1 & 1 \\
\hline
\end{tabular}

$\mathrm{D3}=\mathrm{Q} 3 \mathrm{Q} 0+\mathrm{Q3Q} 2+\overline{\mathrm{Q} 2} \mathrm{Q1} \overline{\mathrm{Q0}}$

\begin{tabular}{|c|c|c|c|c|c|}
\hline D1 & \multicolumn{5}{|c|}{ Q1 Q0 } \\
\hline \multirow{5}{*}{} & & $\mathbf{0 0}$ & $\mathbf{0 1}$ & $\mathbf{1 1}$ & $\mathbf{1 0}$ \\
\cline { 2 - 6 } & $\mathbf{0 0}$ & $\mathrm{X}$ & $\mathrm{X}$ & 1 & 1 \\
\cline { 2 - 6 } & $\mathbf{0 1}$ & 1 & 0 & 1 & 0 \\
\cline { 2 - 6 } Q3Q2 & $\mathbf{1 1}$ & 1 & 0 & $\mathrm{X}$ & 0 \\
\cline { 2 - 6 } & $\mathbf{1 0}$ & 1 & 0 & 1 & 0 \\
\hline
\end{tabular}

$\mathrm{D} 1=\overline{\mathrm{Q3}} \overline{\mathrm{Q} 2}+\overline{\mathrm{Q} 1} \overline{\mathrm{Q} 0}+\mathrm{Q} 1 \mathrm{Q0}$

$$
\begin{aligned}
\mathrm{D} 3 & =\mathrm{Q} 3 \mathrm{Q} 0+\mathrm{Q} 3 \mathrm{Q} 2+\overline{\mathrm{Q} 2} \mathrm{Q} 1 \overline{\mathrm{Q} 0} \\
& =\mathrm{Q} 3(\mathrm{Q} 0+\mathrm{Q} 2)+\mathrm{Q} 1 \overline{(\mathrm{Q} 0+\mathrm{Q} 2}) \\
& =\overline{\mathrm{Q} 3(\mathrm{Q} 0+\mathrm{Q} 2)+\mathrm{Q} 1 \overline{(\overline{\mathrm{Q} 0+\mathrm{Q} 2})}}
\end{aligned}
$$

$$
\begin{aligned}
& D 2=Q 2 \cdot Q 0+Q 2 \cdot Q 1+\overline{Q 3} \cdot Q 1 \cdot \overline{Q 0}+\overline{Q 2} \cdot \overline{Q 1} \cdot \overline{Q 0}= \\
& =Q 2 \cdot(Q 0+Q 1)+\overline{Q 3} \cdot Q 1 \cdot \overline{Q 0}+\overline{Q 2} \cdot \overline{\overline{Q 1} \cdot \overline{Q 0}}= \\
& =Q 2 \cdot(Q 1+Q 0)+\overline{Q 3} \cdot Q 1 \cdot \overline{Q 0}+\overline{Q 2} \cdot \overline{(Q 1+Q 0)}
\end{aligned}
$$

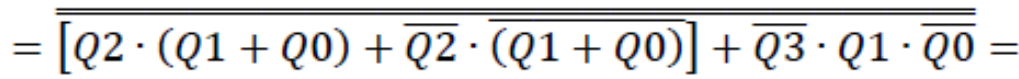

$$
\begin{aligned}
& =\overline{[Q 2 \cdot \overline{(Q 1+Q 0)}+\overline{Q 2} \cdot(Q 1+Q 0)] \cdot \overline{\overline{Q 3} \cdot Q 1 \cdot \overline{Q 0}}}
\end{aligned}
$$

\begin{tabular}{|c|c|c|c|c|c|}
\hline D0 & \multicolumn{5}{|c|}{ Q1 Q0 } \\
\hline \multirow{5}{*}{ Q3Q2 } & & $\mathbf{0 0}$ & $\mathbf{0 1}$ & $\mathbf{1 1}$ & $\mathbf{1 0}$ \\
\cline { 2 - 7 } & $\mathbf{0 0}$ & $\mathrm{X}$ & $\mathrm{X}$ & 0 & 0 \\
\cline { 2 - 6 } & $\mathbf{0 1}$ & 1 & 0 & 0 & 1 \\
\cline { 2 - 6 } & $\mathbf{1 1}$ & 1 & 0 & $\mathrm{X}$ & 1 \\
\cline { 2 - 6 } & $\mathbf{1 0}$ & 1 & 0 & 0 & 1 \\
\hline
\end{tabular}

$\mathrm{D} 0=\mathrm{Q} 2 \overline{\mathrm{Q0}}+\mathrm{Q3} \overline{\mathrm{Q0}}$

$$
=\overline{\overline{\mathrm{Q} 3}+\overline{\mathrm{Q} 0} \overline{\mathrm{Q} 2} \cdot \overline{\mathrm{Q} 1 \overline{(\overline{\mathrm{Q} 0+\mathrm{Q} 2})}}}
$$




$$
\begin{aligned}
& \mathrm{D} 1=\overline{\mathrm{Q} 3} \overline{\mathrm{Q} 2}+\overline{\mathrm{Q} 1} \overline{\mathrm{Q} 0}+\mathrm{Q} 1 \mathrm{Q} 0 \\
& =\overline{\mathrm{Q} 3} \overline{\mathrm{Q} 2}+(\mathrm{Q} 1 \odot \mathrm{Q} 0)=\overline{\overline{\mathrm{Q} 3} \overline{\mathrm{Q} 2}+(\mathrm{Q} 1 \odot \mathrm{Q} 0)} \\
& \mathrm{D} 1=\overline{(\mathrm{Q} 3+\mathrm{Q} 2) \cdot(\overline{\mathrm{Q} 1} \oplus \overline{\mathrm{Q} 0})} \\
& \mathrm{D} 0=\mathrm{Q} 2 \overline{\mathrm{Q} 0}+\mathrm{Q} 3 \overline{\mathrm{Q} 0} \quad=\overline{\overline{\overline{\mathrm{Q} 0}(\mathrm{Q} 2+\mathrm{Q} 3)}} \\
& \mathrm{D} 0=\overline{\mathrm{Q} 0+\overline{\mathrm{Q} 2 .} \overline{\mathrm{Q} 3}}
\end{aligned}
$$

\subsection{Implementation on circuit level}

The block diagram of the MOD 13 counter is provided in fig. 2.1, all the inputs (D0, D1, D2, and D3) are realized on circuit level (using only logic gates), then in section 2.4, CMOS implementation of all the used logic gates are provided.

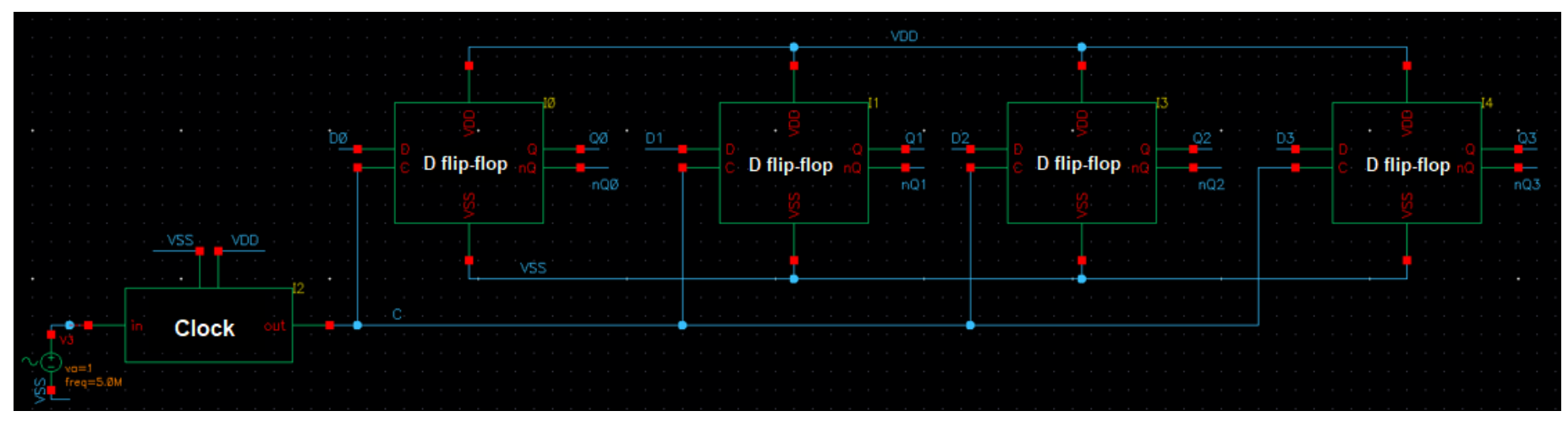

Fig 2.1 MOD 13 counter (block diagram) 


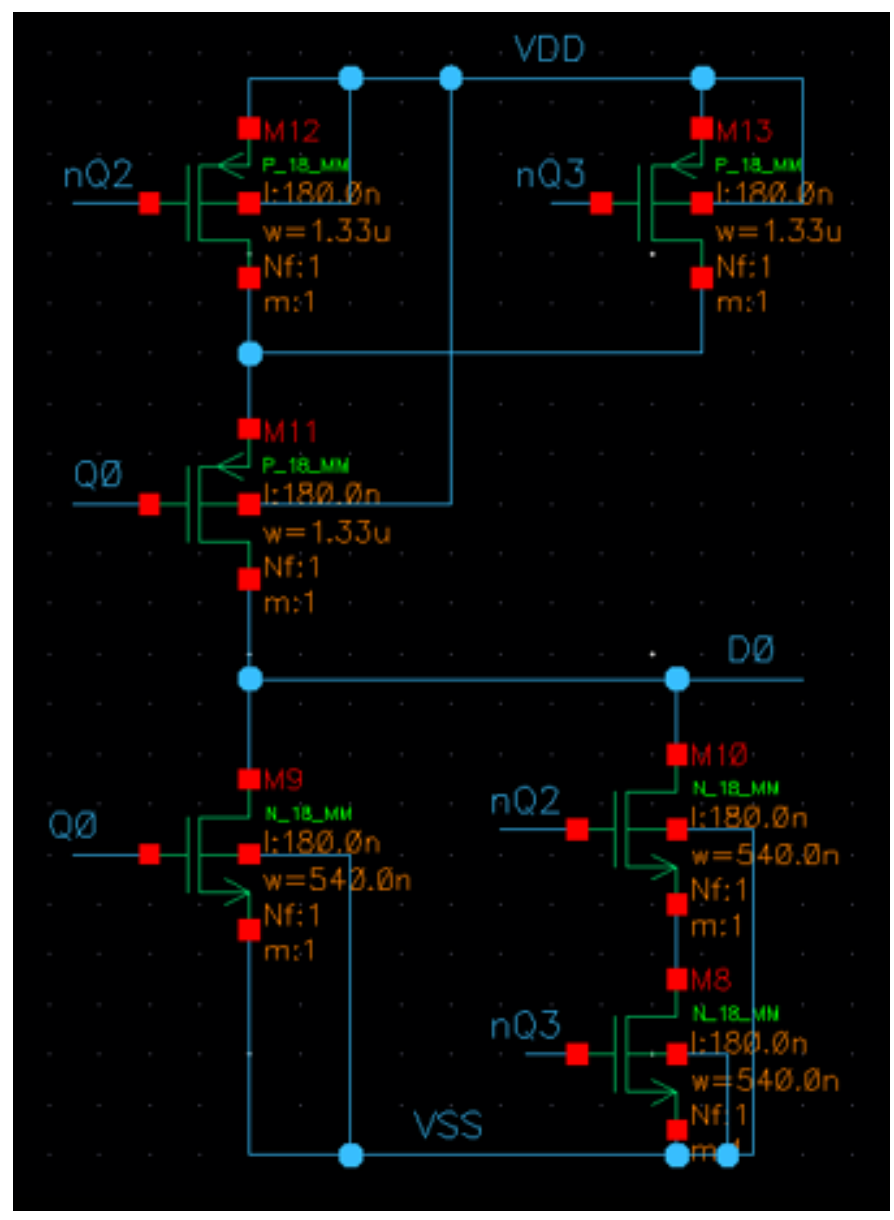

Fig 2.2 Input D0

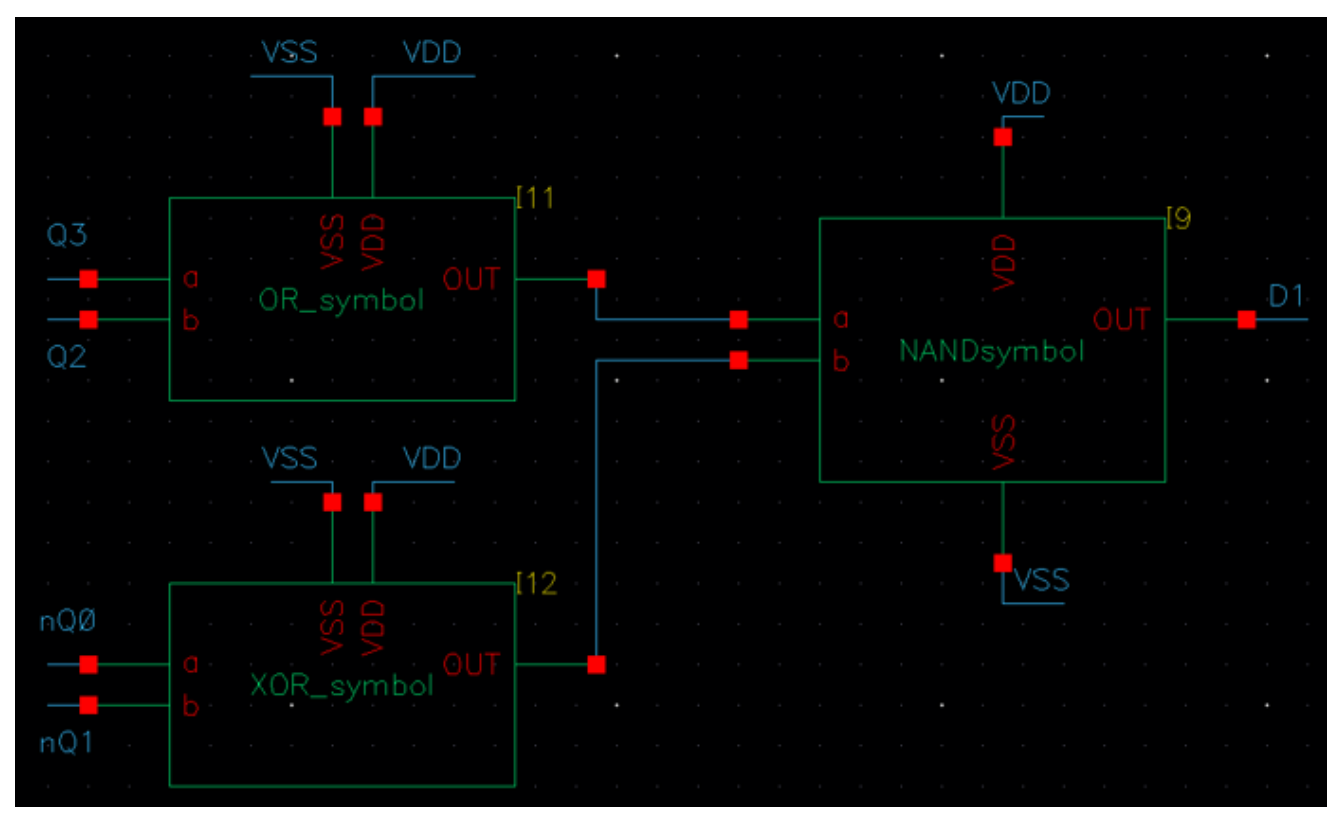

Fig 2.3 Input D1 


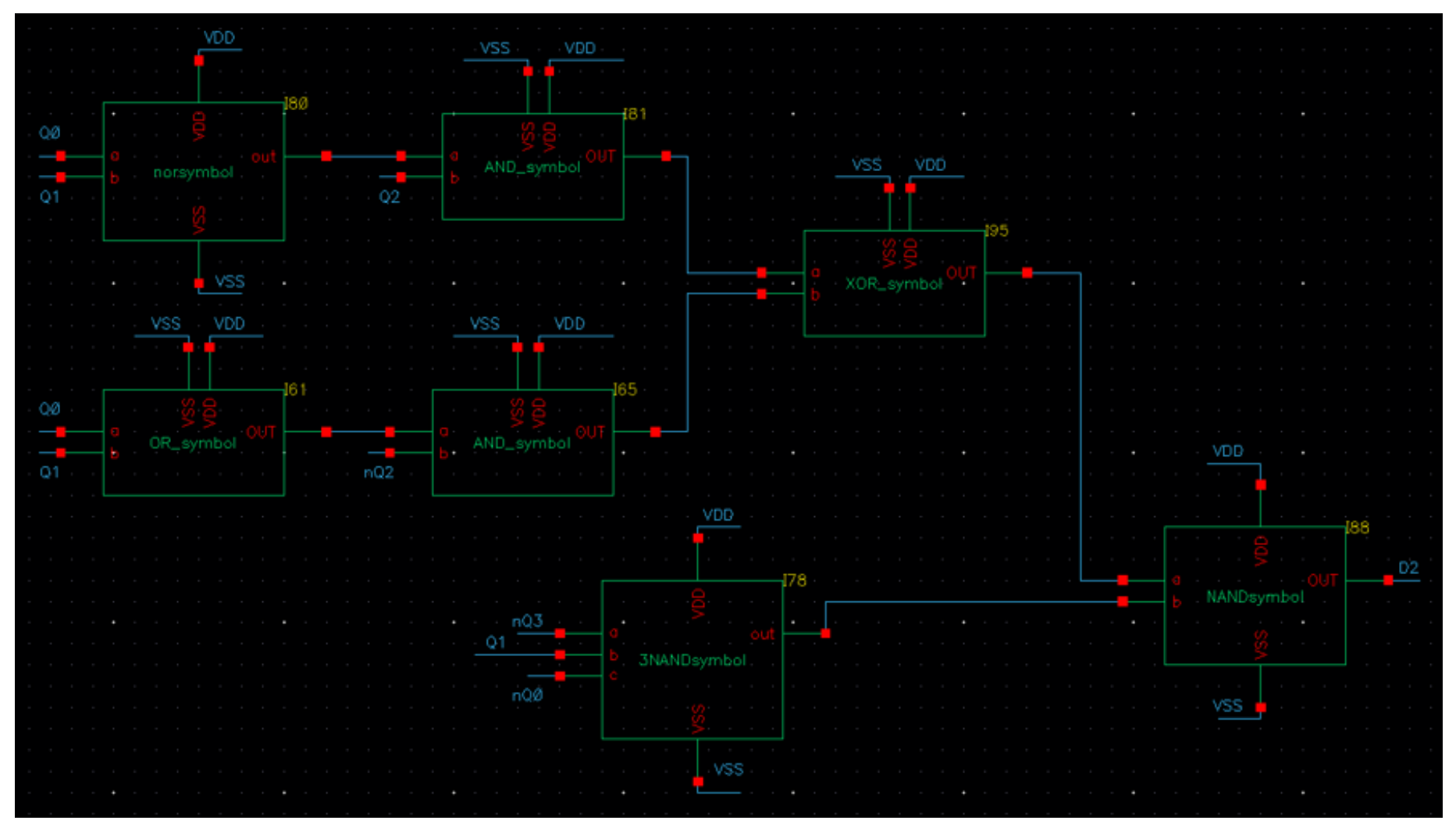

Fig 2.4 Input D2

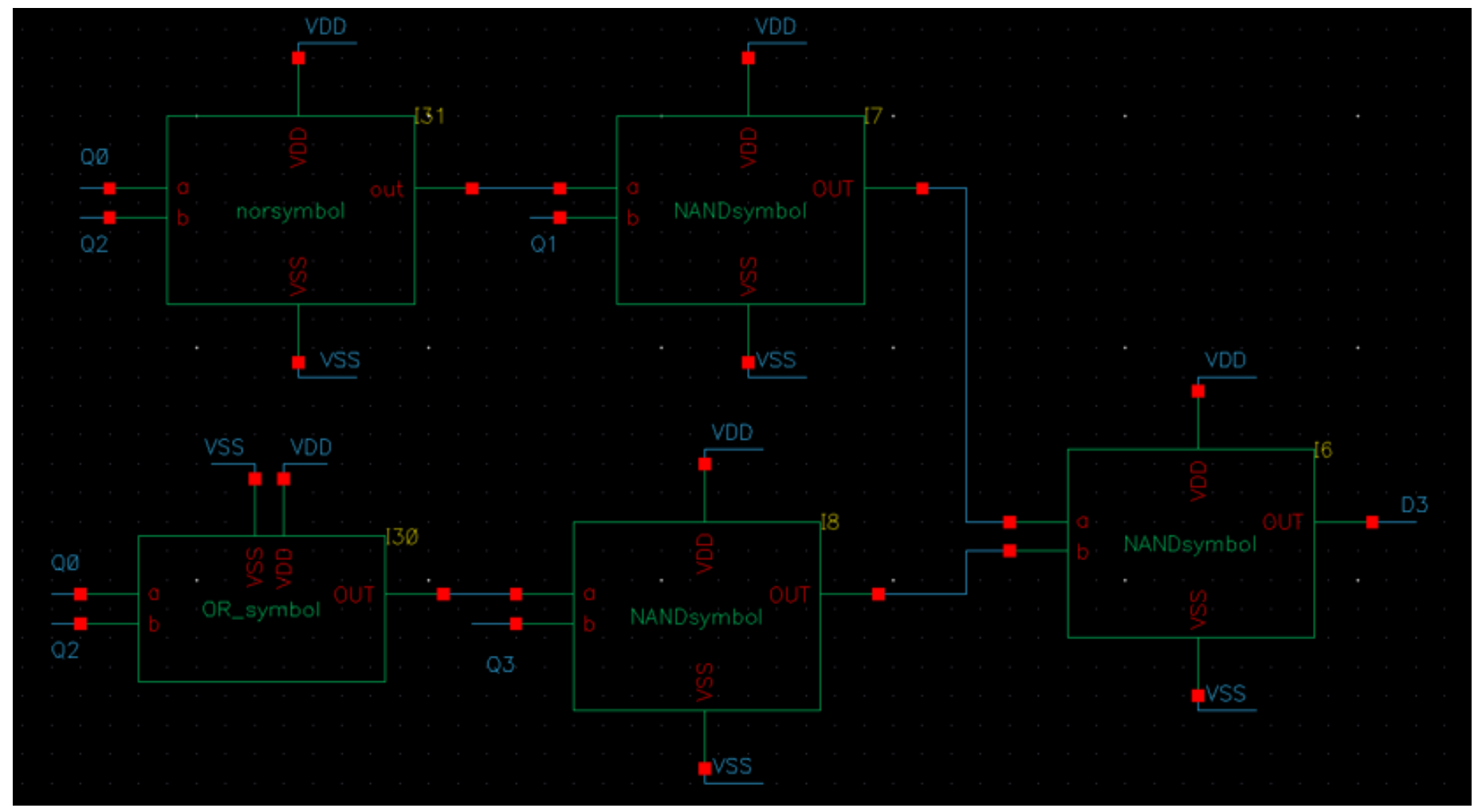

Fig 2.5 Input D3 
2.4 Implementation on transistor level

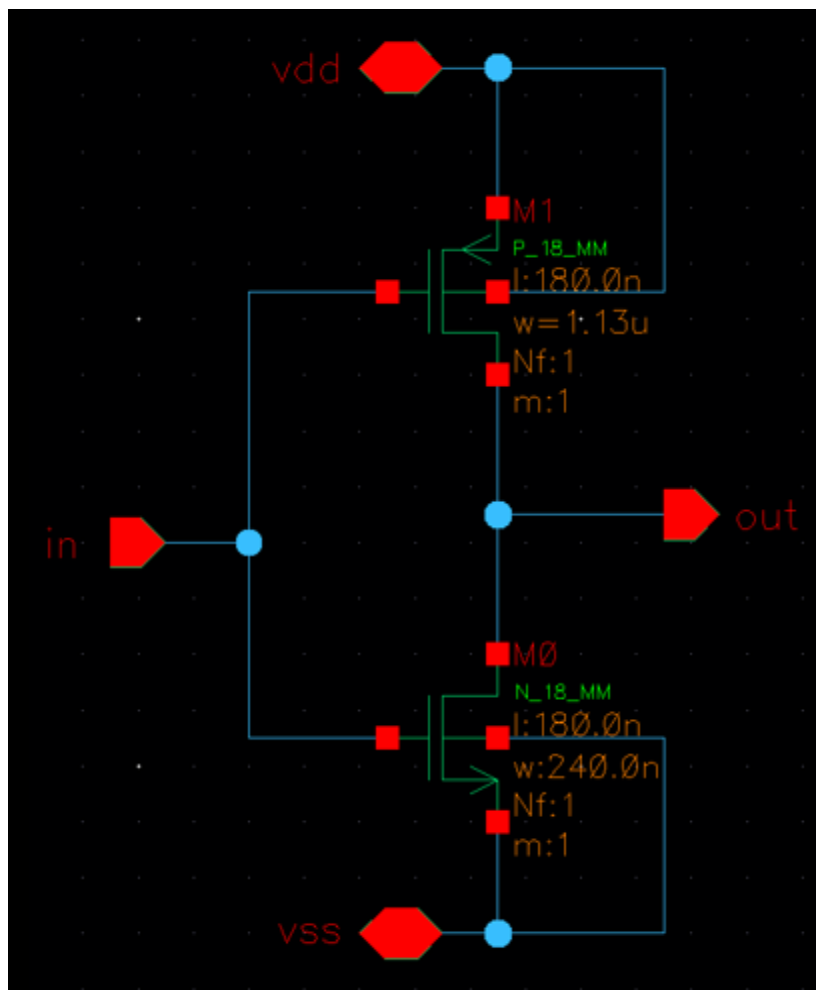

Fig 2.6 Inverter schematic

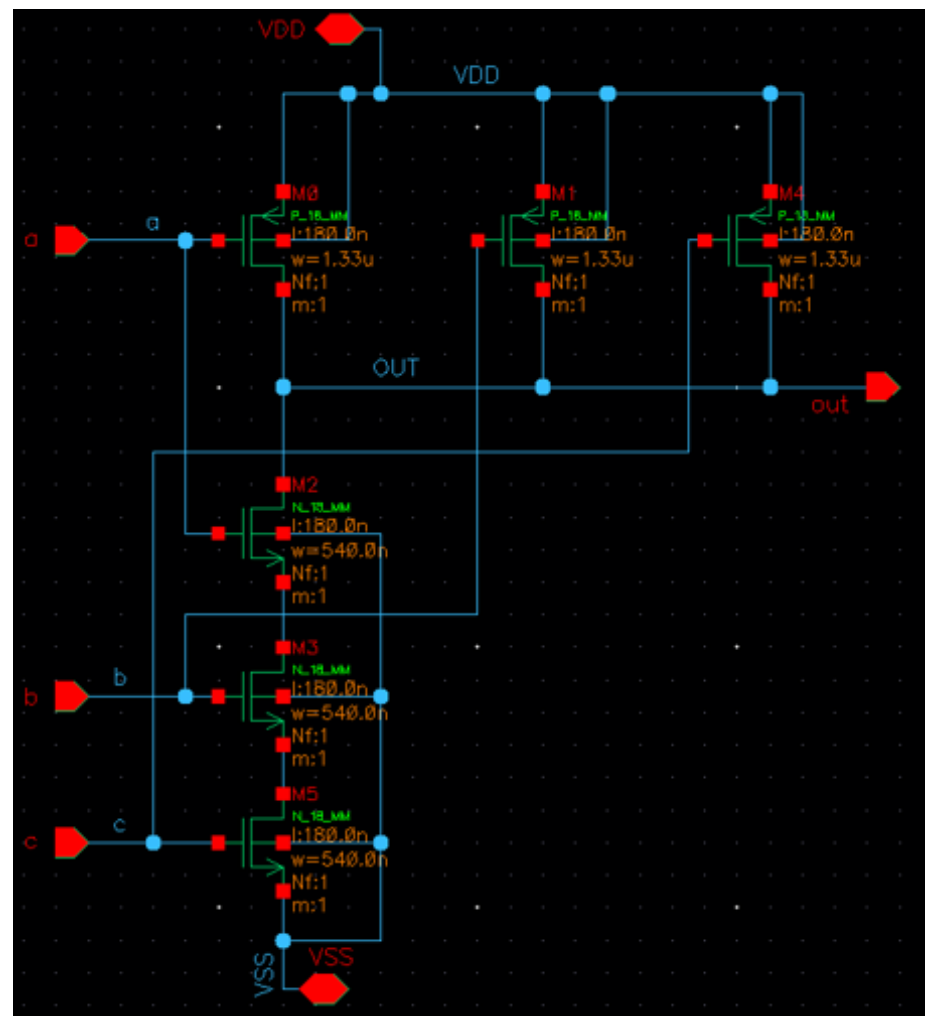

Fig 2.7 NAND gate (3 inputs) 


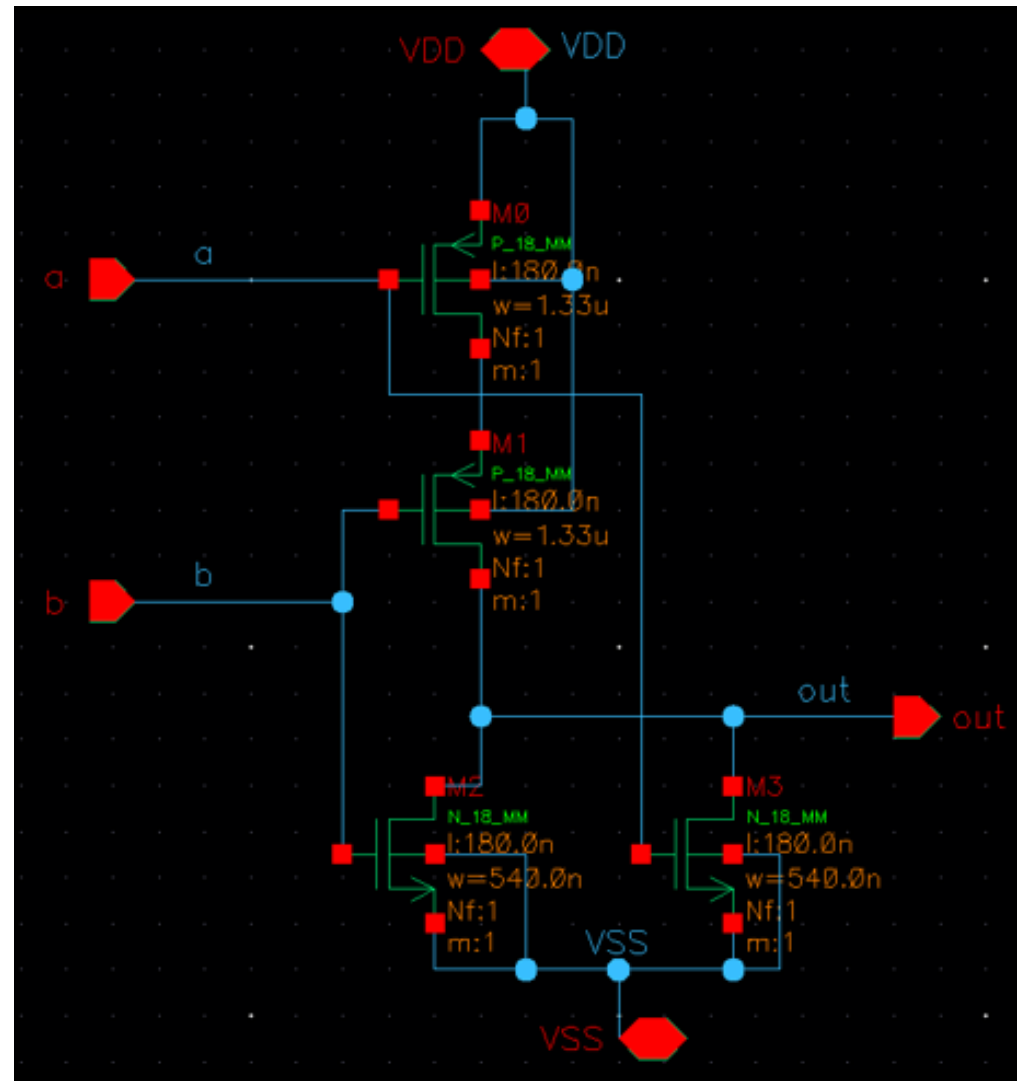

Fig 2.8 NOR gate with 2 inputs

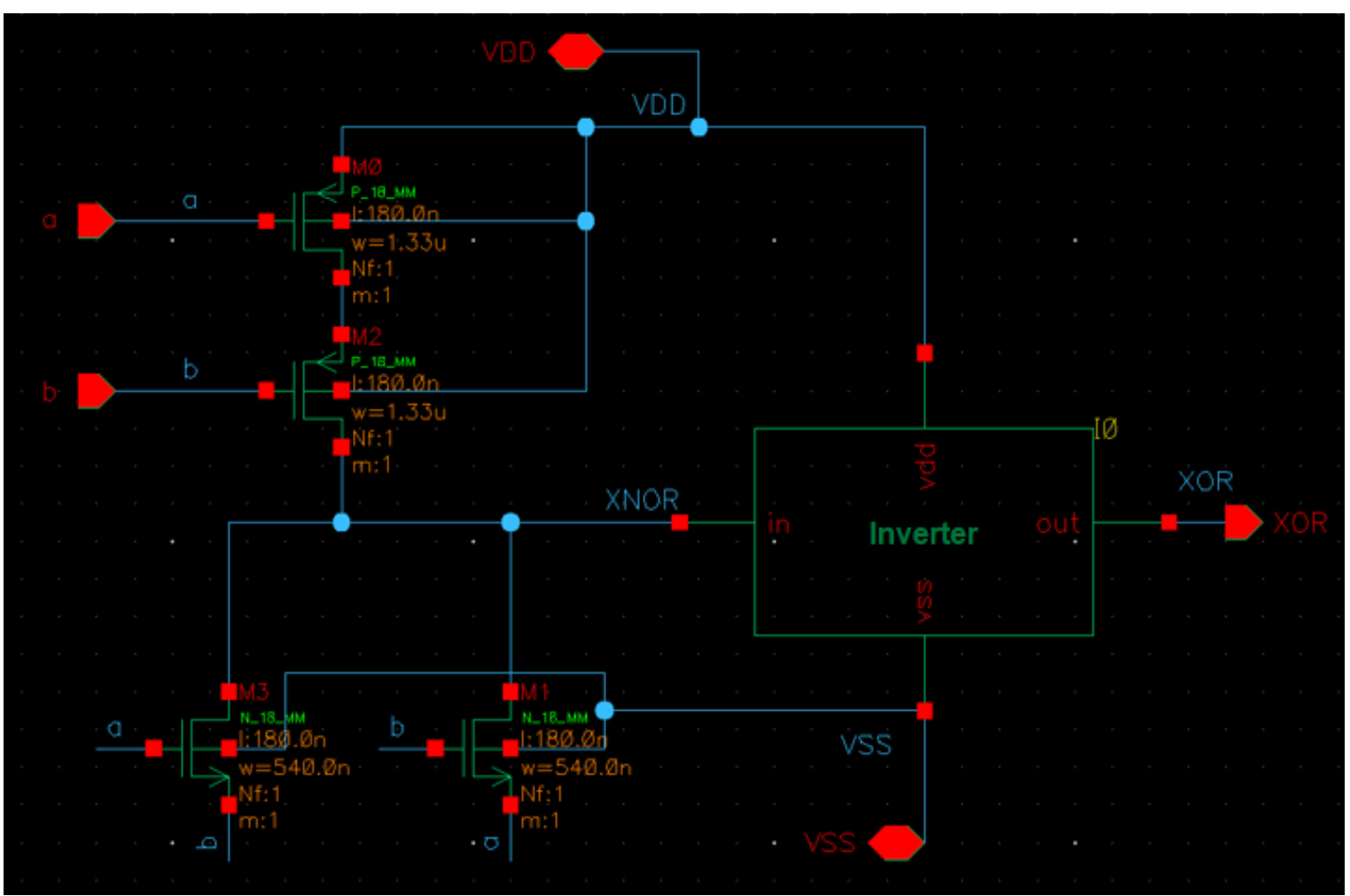

Fig 2.9 Exclusive OR gate 


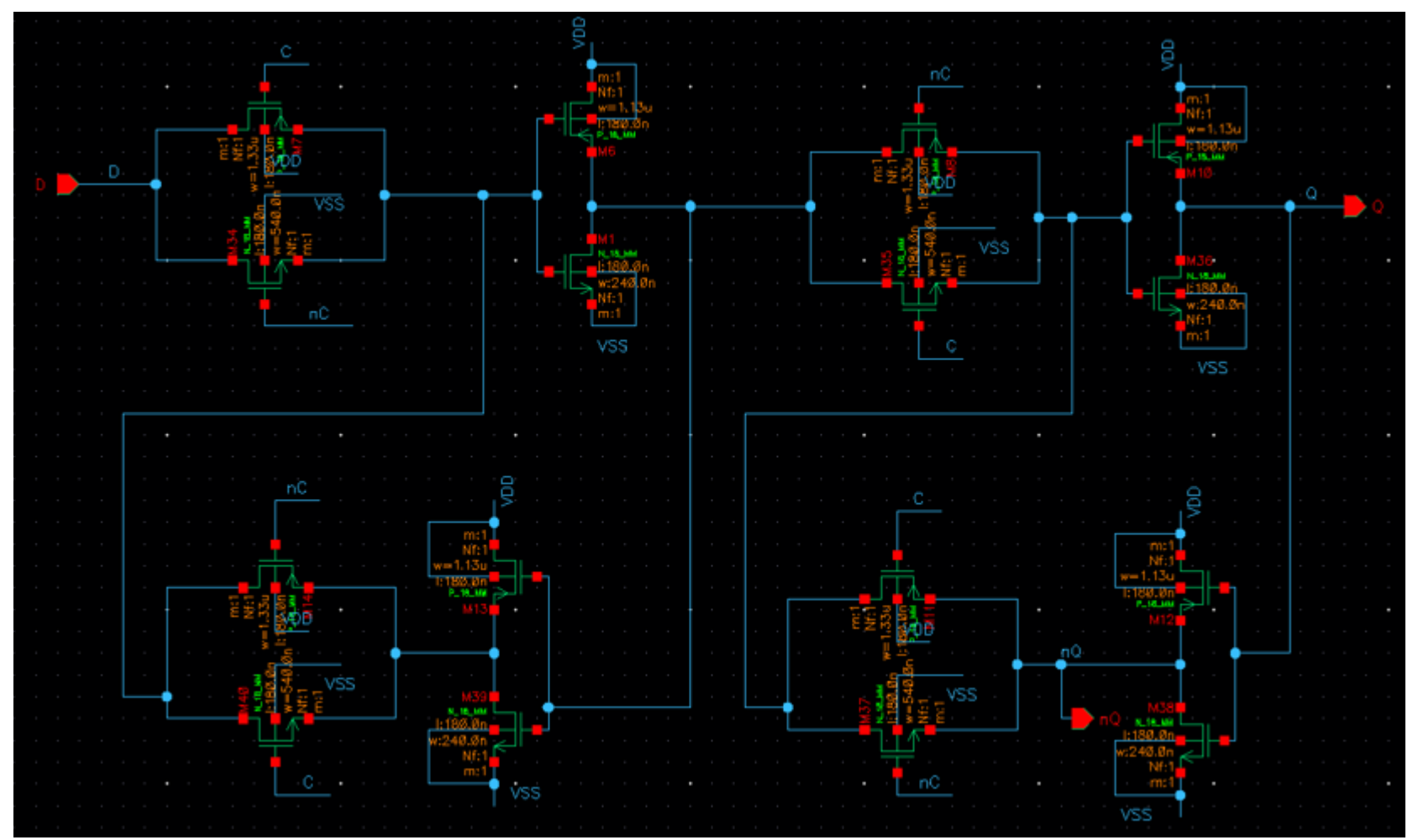

Fig 2.10 D - trigger schematic

\subsection{Results}

After running the simulation (LVS) the following output signals were obtained:

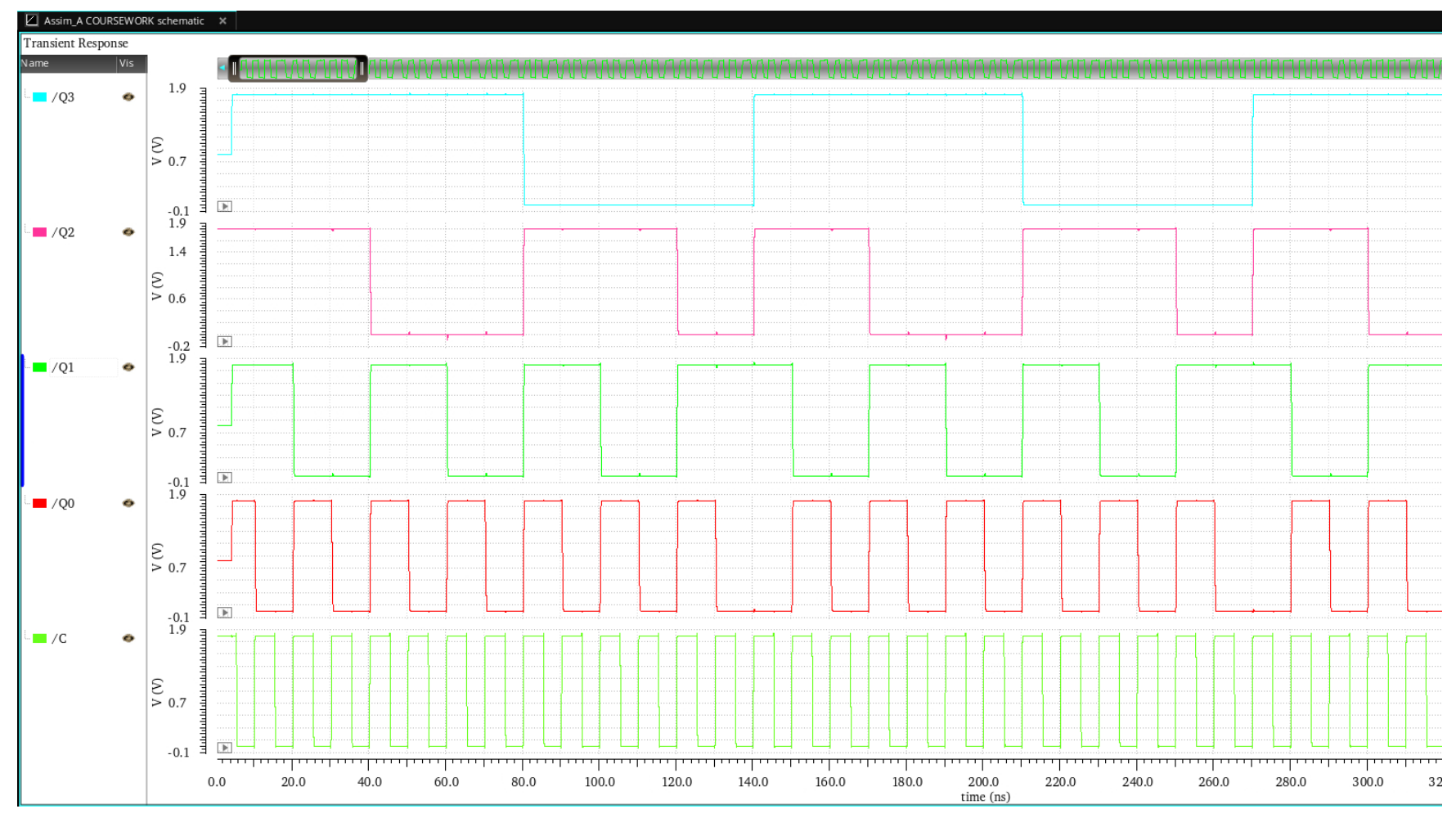

Fig 2.11 Results at clock frequency $=100 \mathrm{MHz}$ 


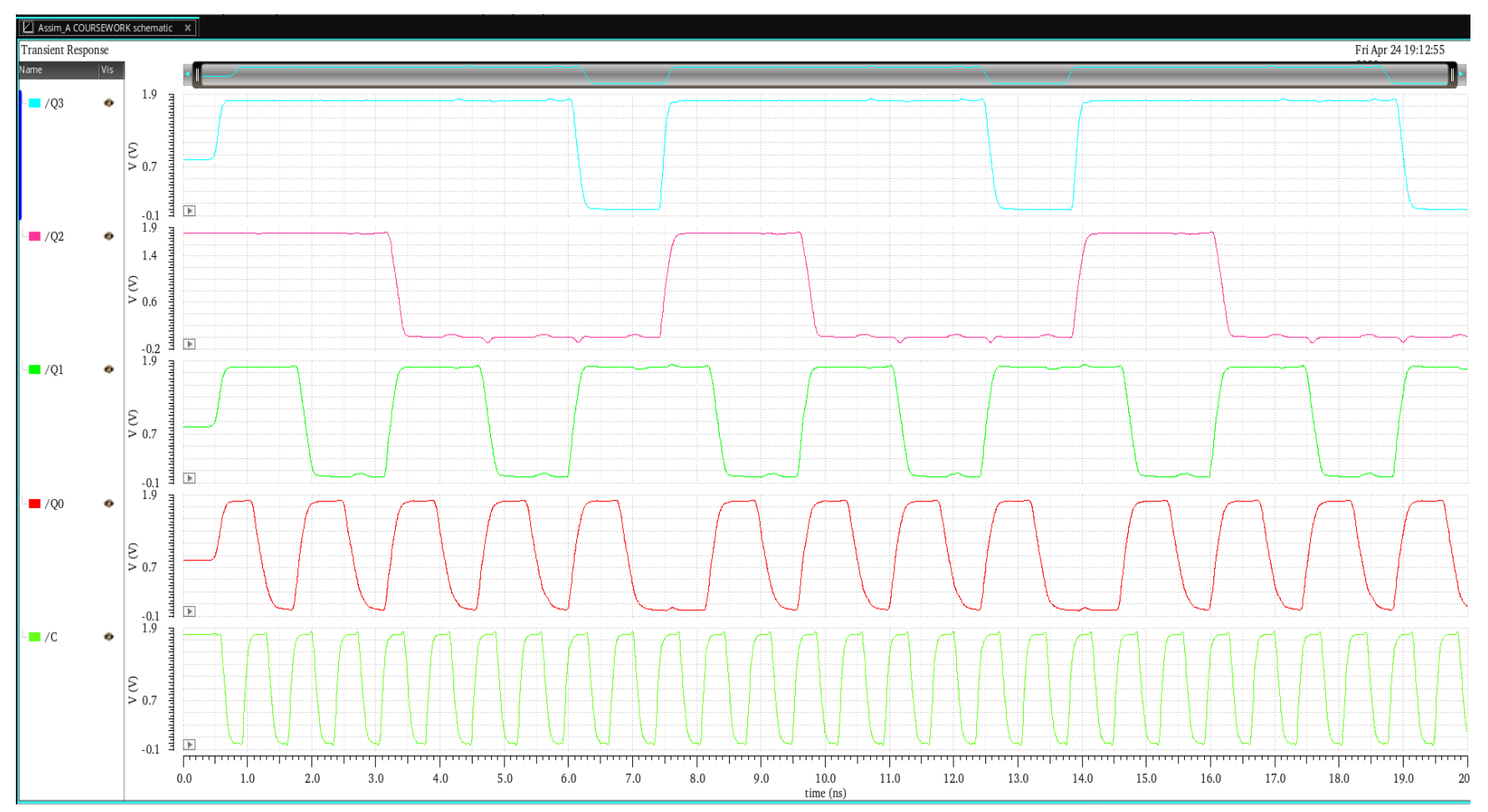

Fig 2.12 Results at clock frequency $=1.4 \mathrm{GHz}$

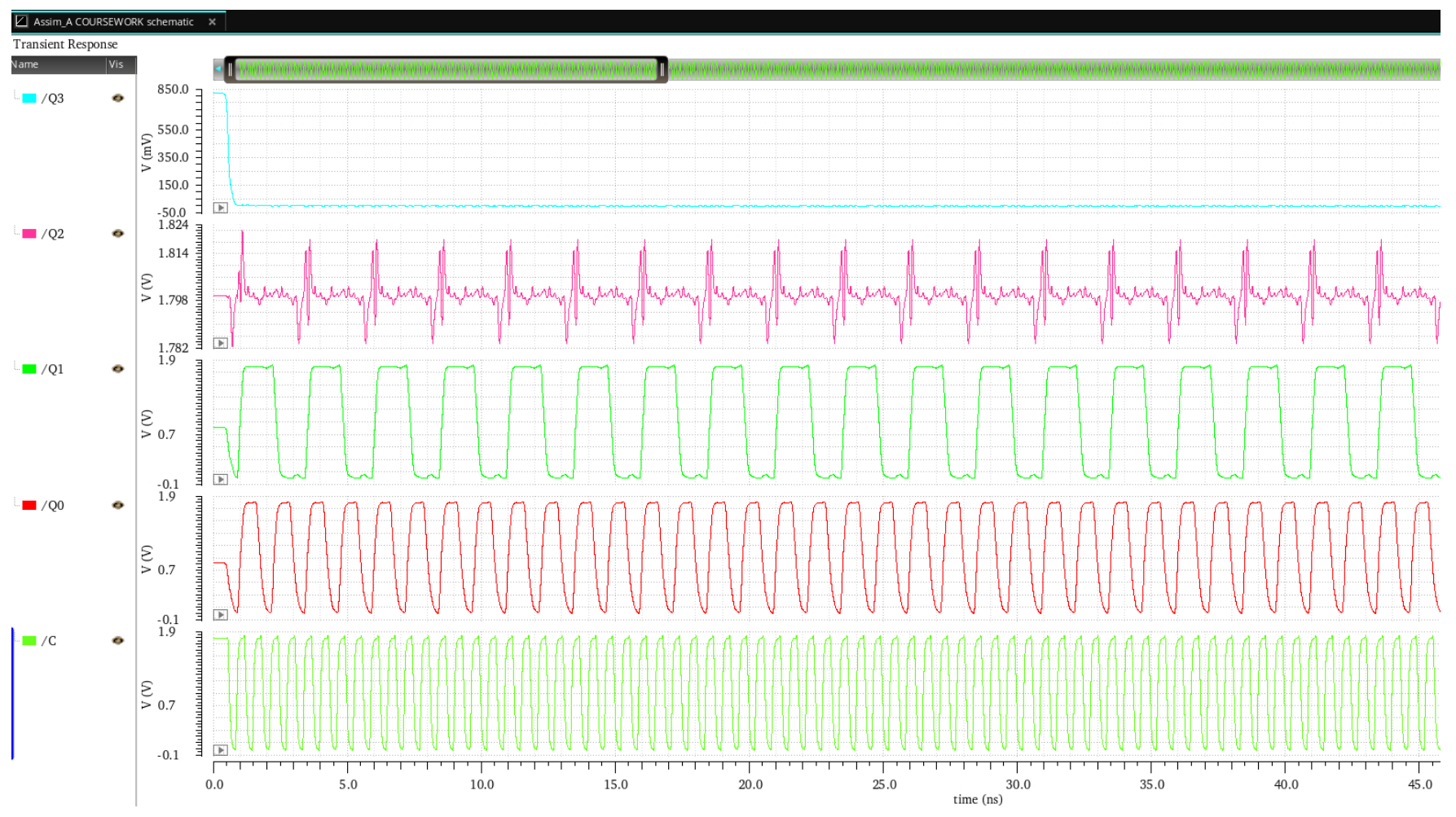

Fig 2.13 Results at clock frequency $=1.6 \mathrm{GHz}$ 


\subsection{Layout design}

In section 2.6, the layouts are prepared for the previously proposed circuits. Layout is created for each element separately, then they are connected through metals.

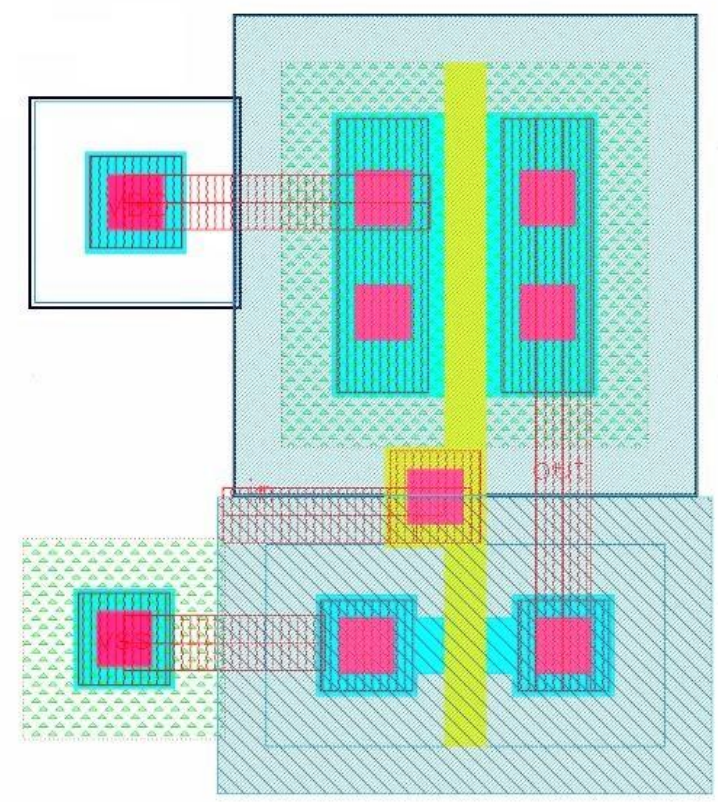

Fig 2.14 Inverter layout

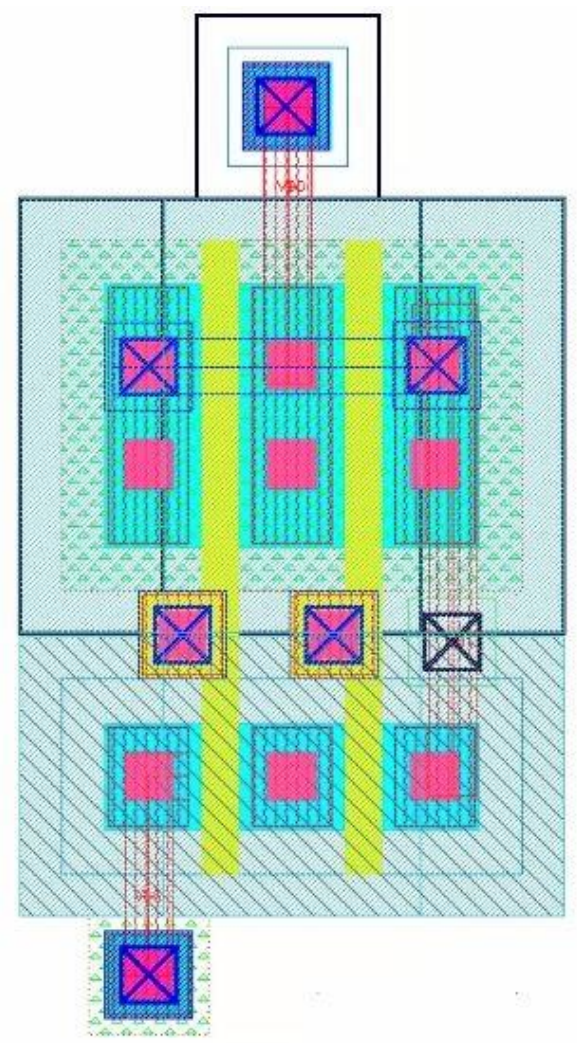

Fig 2.15 NAND2 layout 


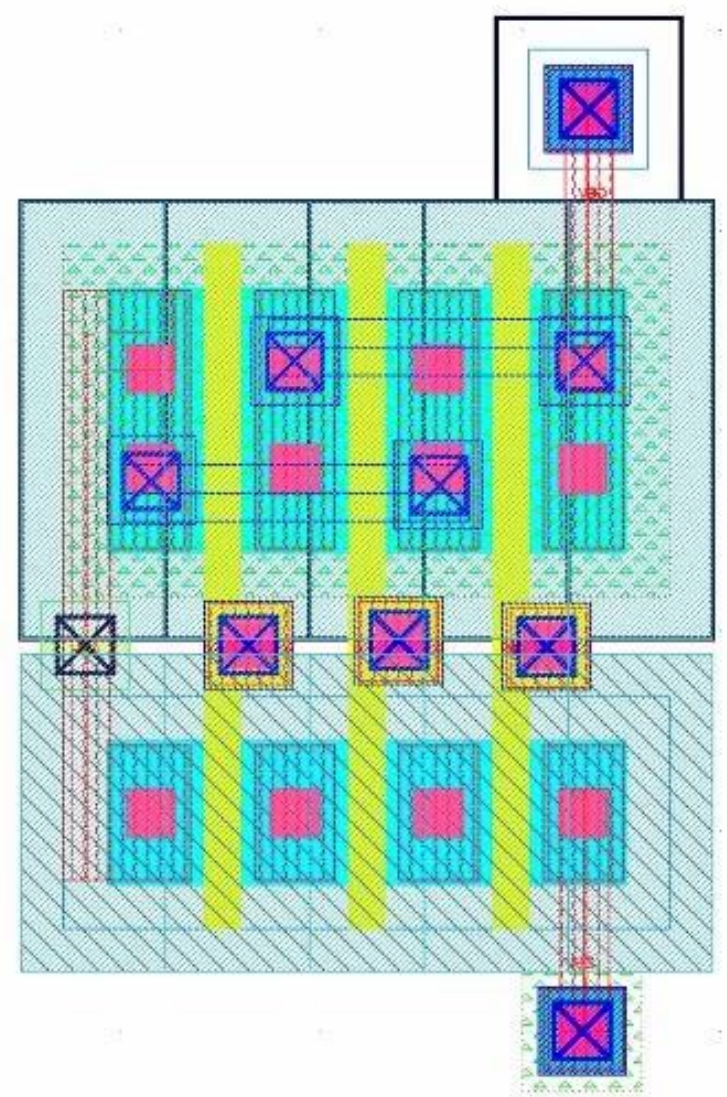

Fig 2.16 NAND3 layout

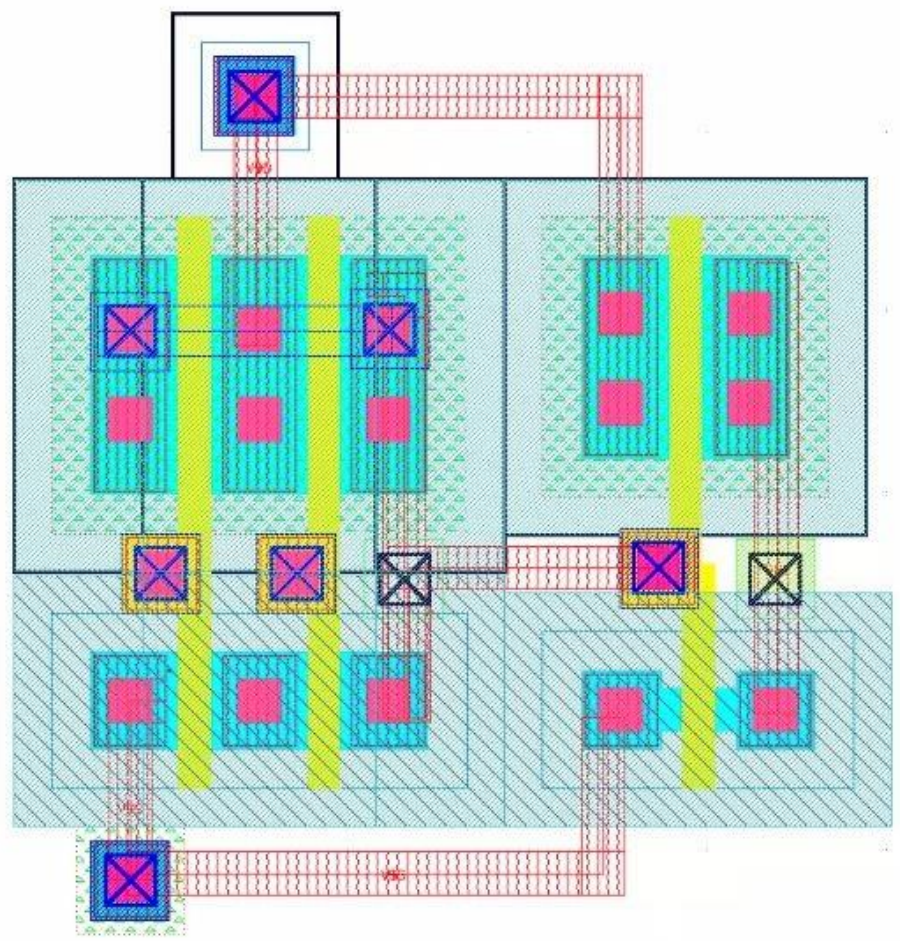

Fig 2.17 AND2 layout 


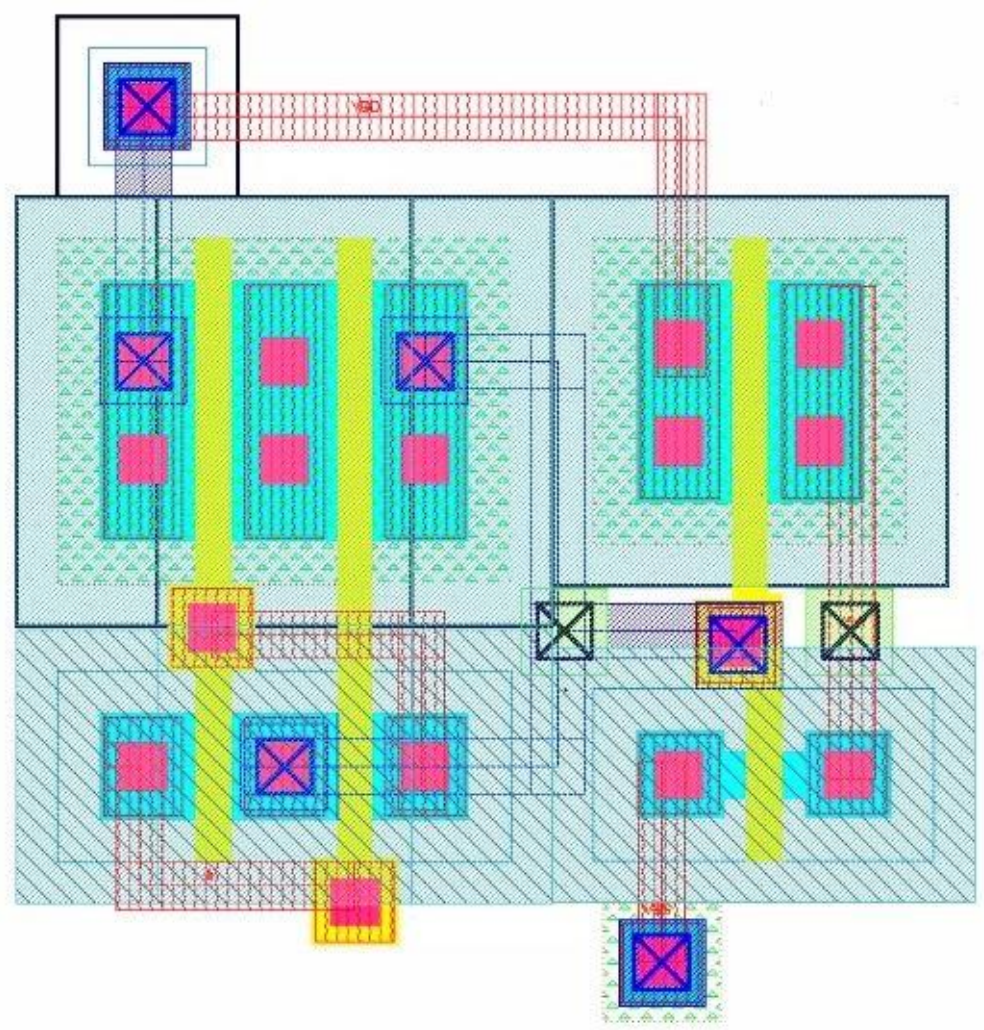

Fig 2.18 XOR layout

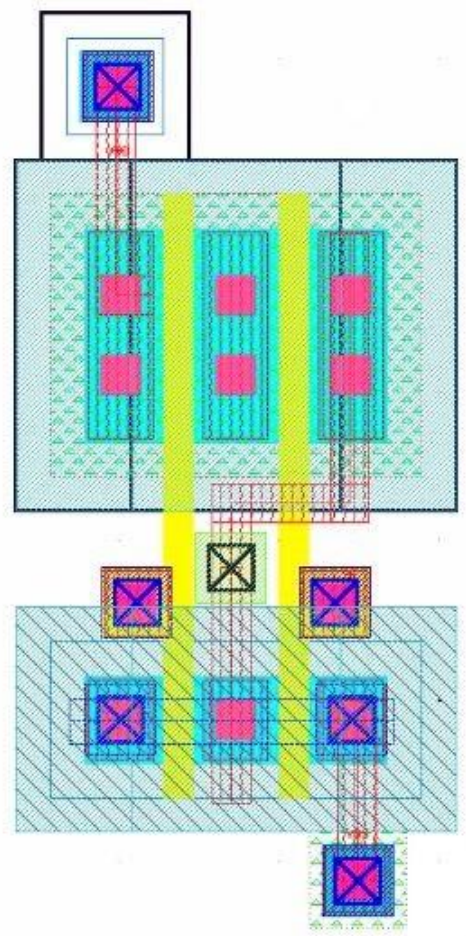

Fig 2.19 NOR2 layout 


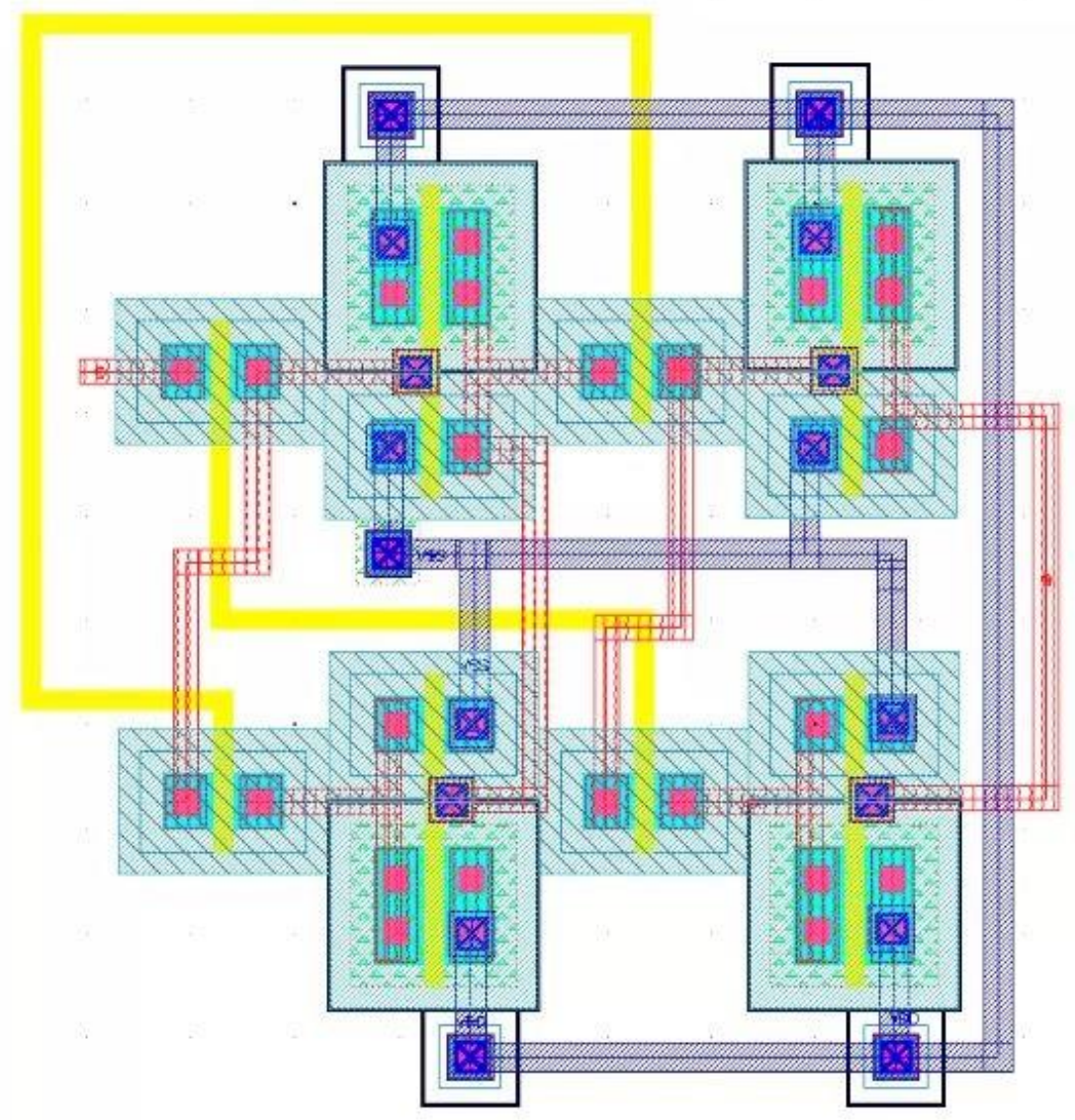

Fig 2.20 D-trigger layout

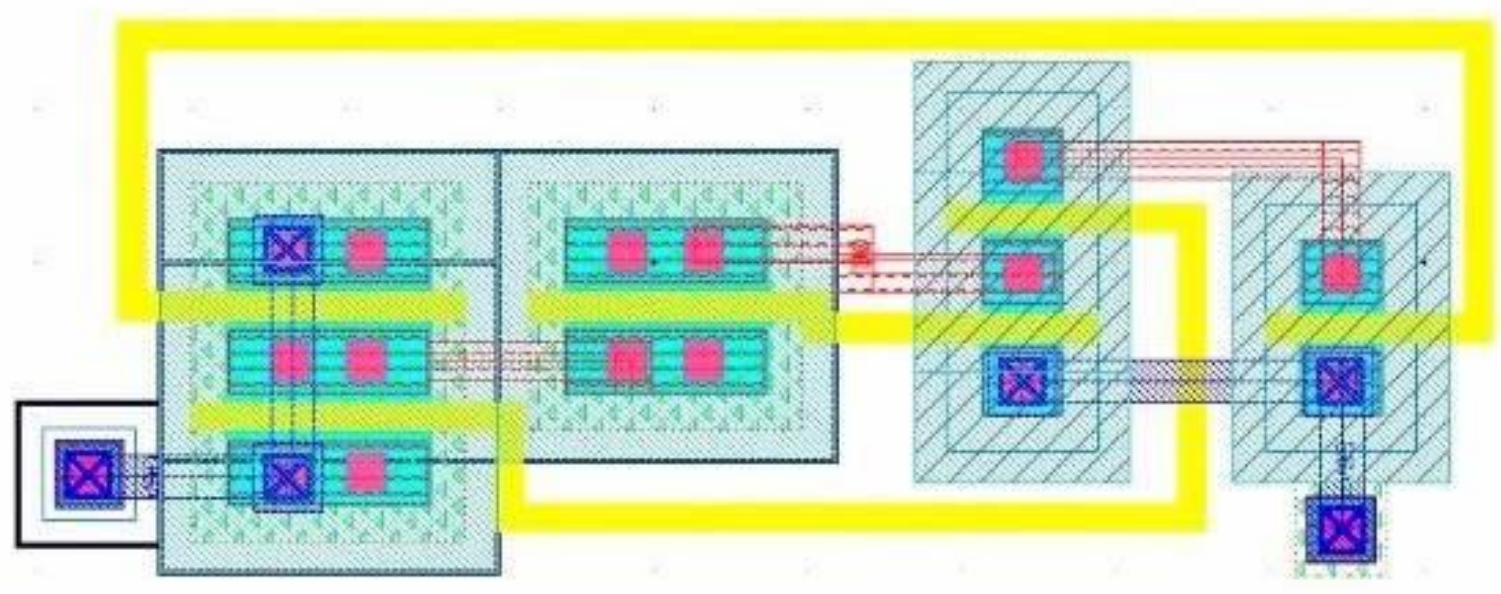

Fig 2.21 Layout of output D0 


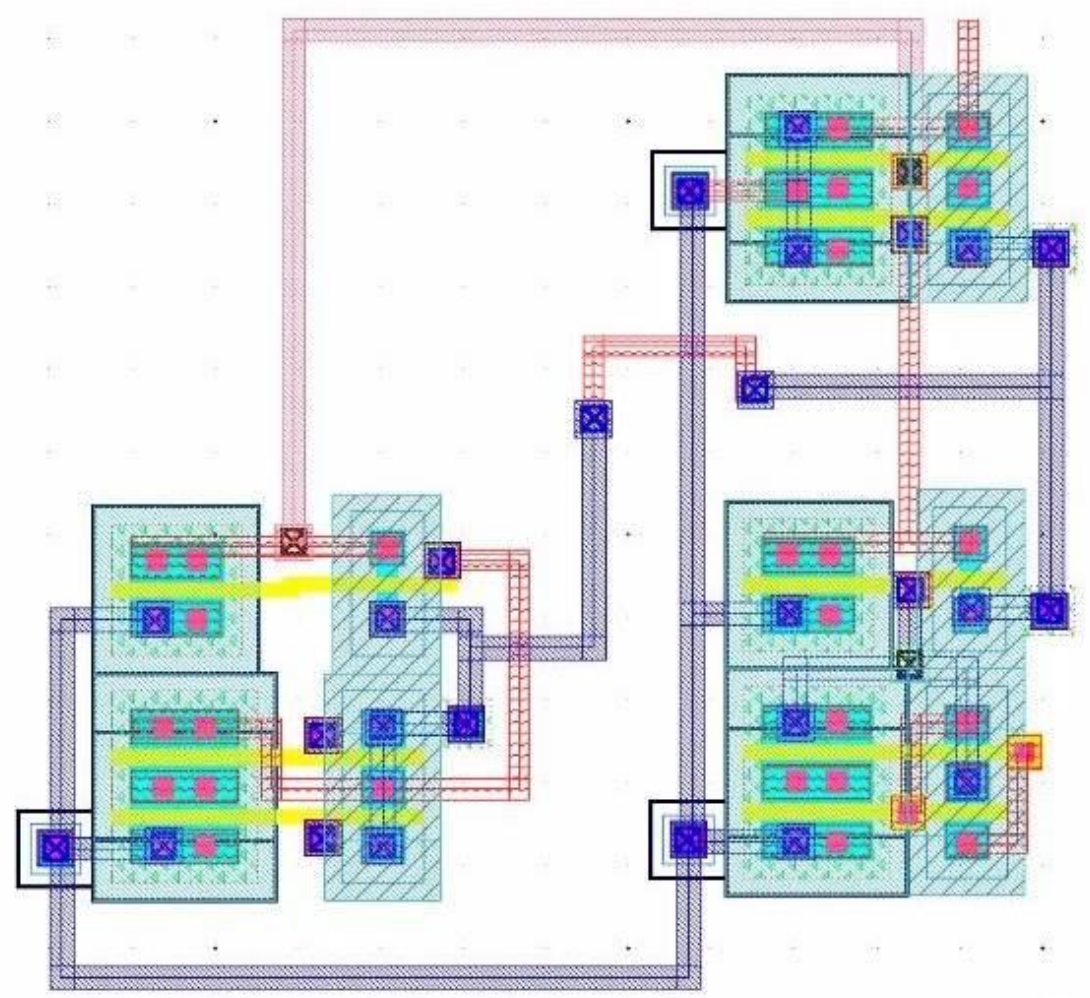

Fig 2.22 Layout of output D1

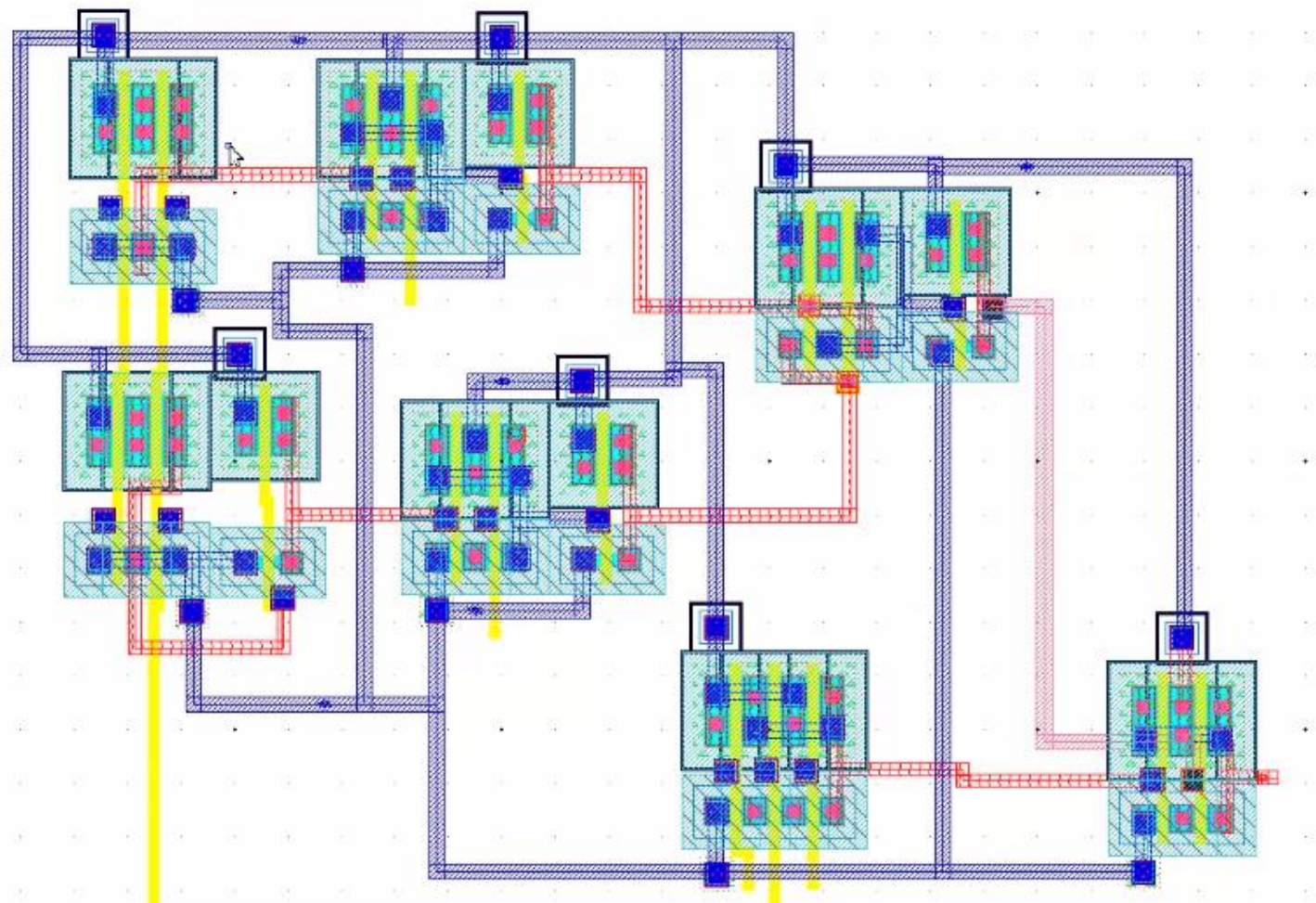

Fig 2.23 Layout of output D2 


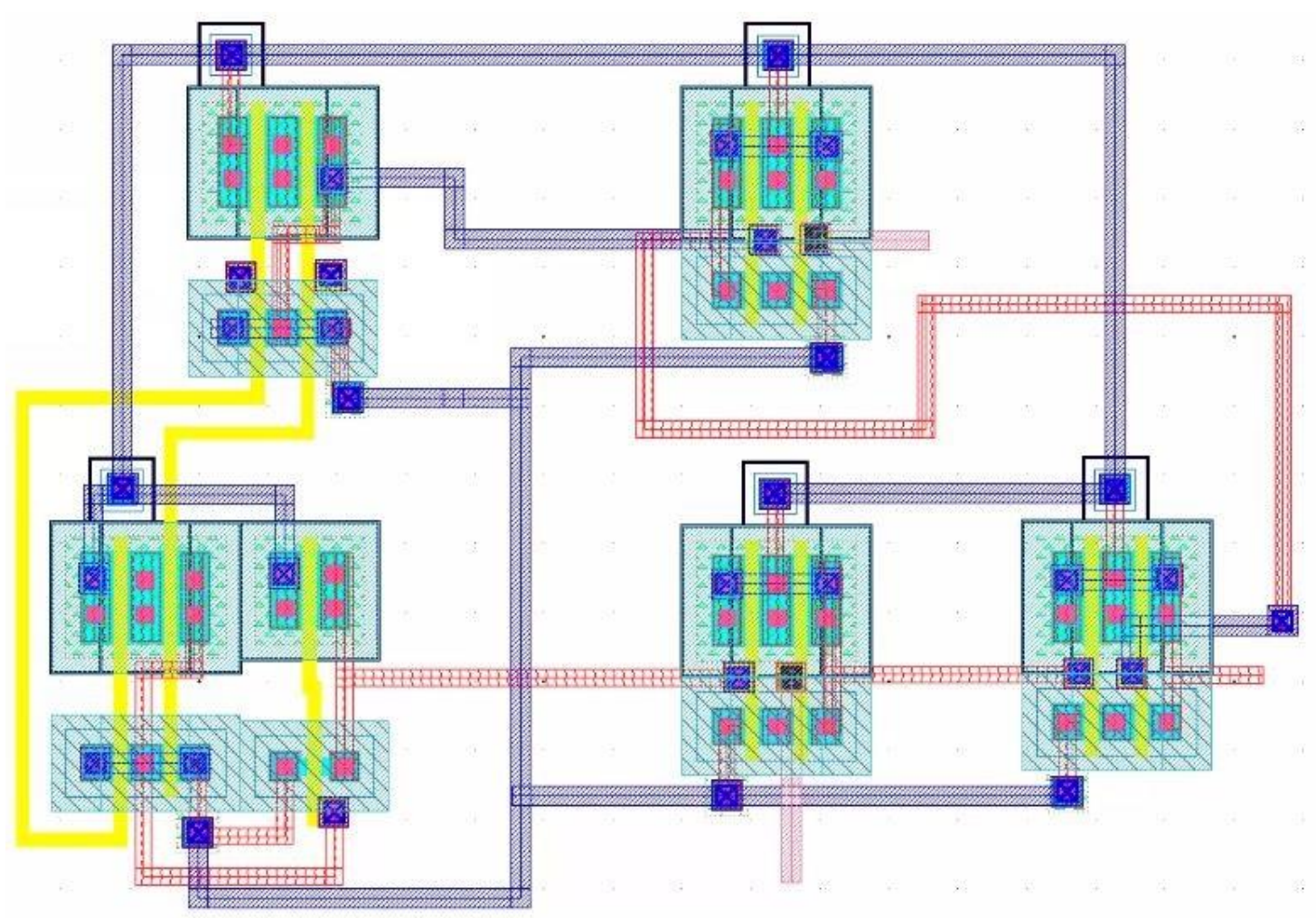

Fig 2.24 Layout f output D3

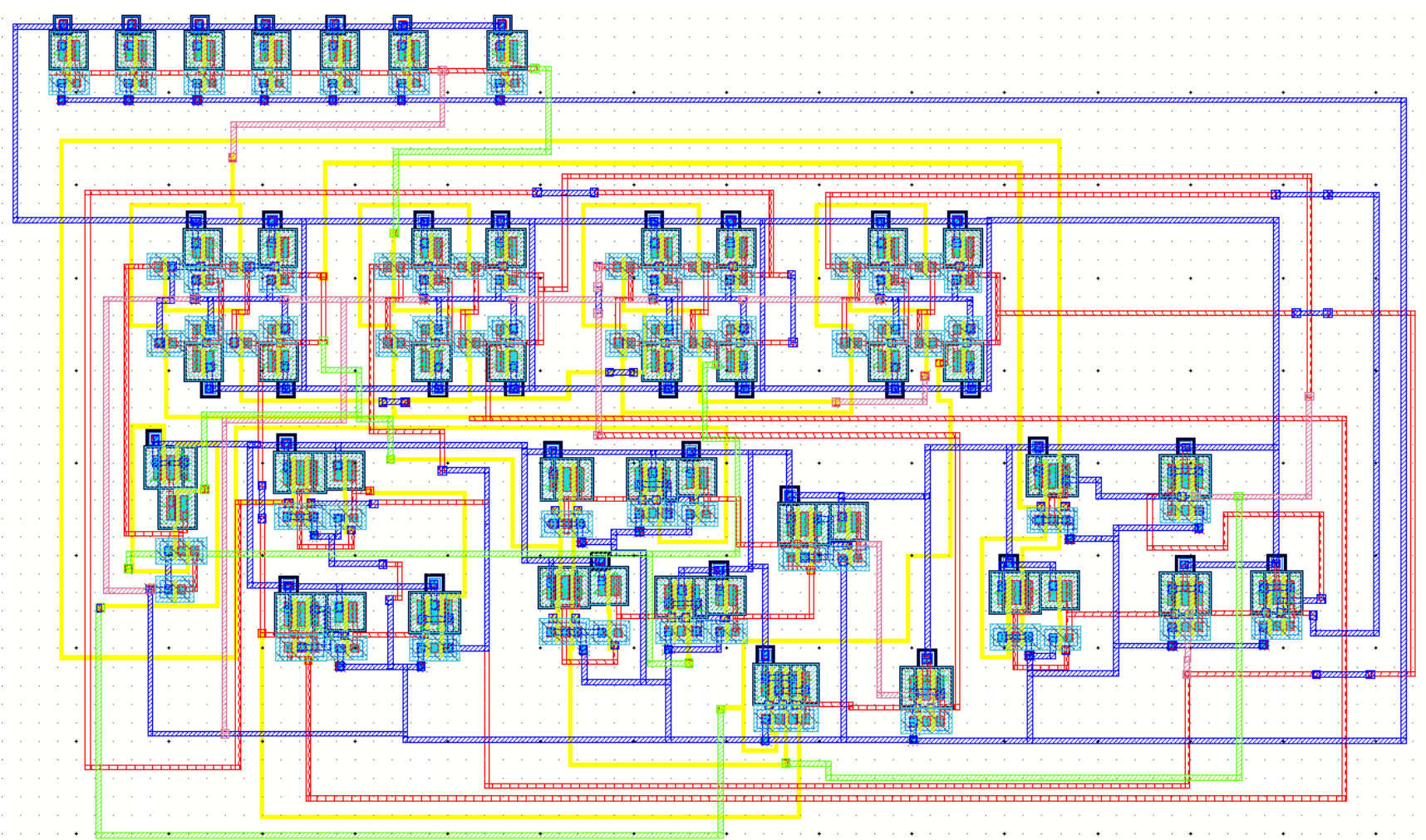

Fig 2.25 Layout of the counter 
Figure 2.25 shows the complete layout of the MOD 13 counter, we may now run DRC and LVS checks to ensure everything works fine and there are no design violations.
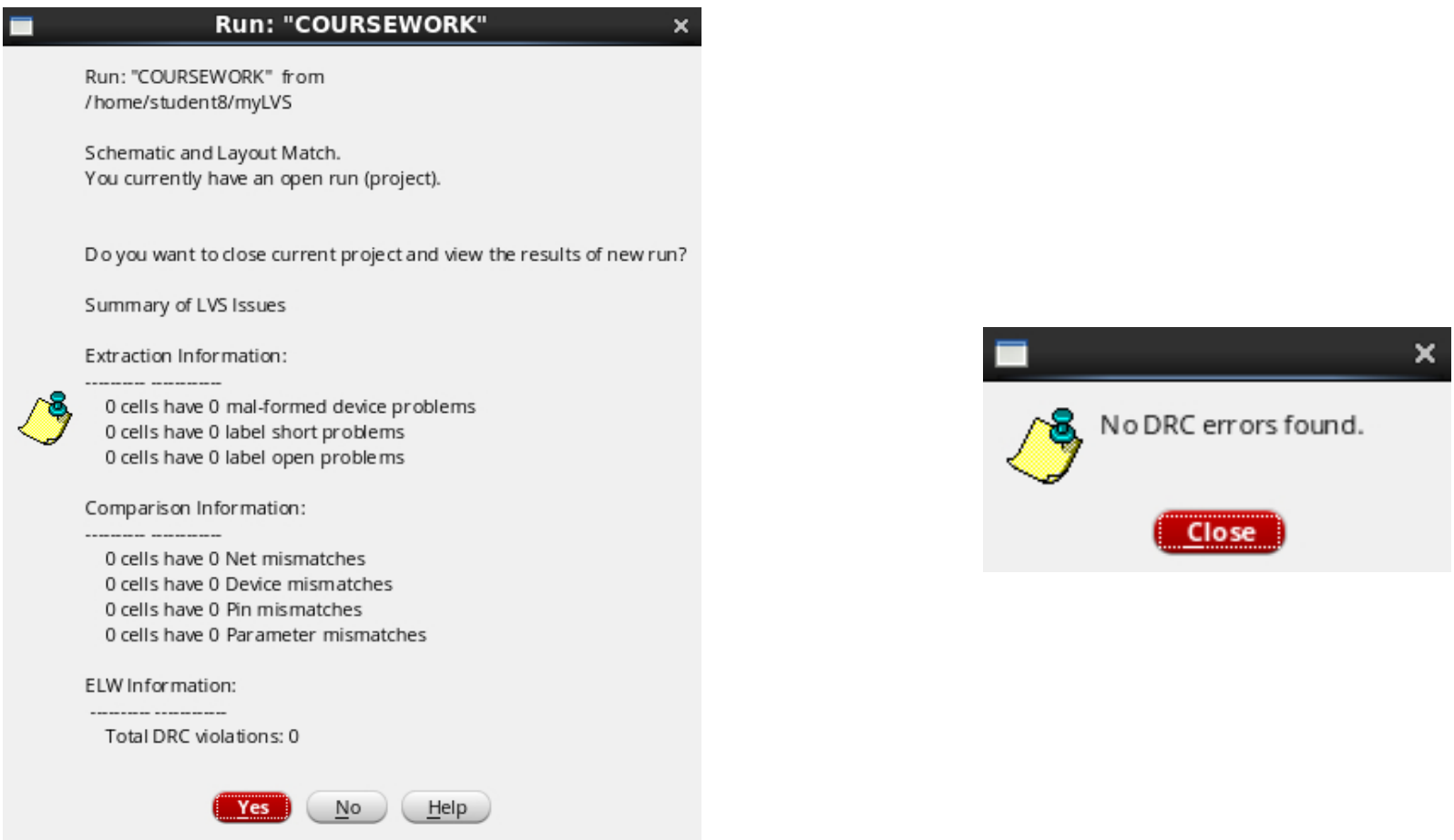

Fig 2.26 Results of DRC and LVS run

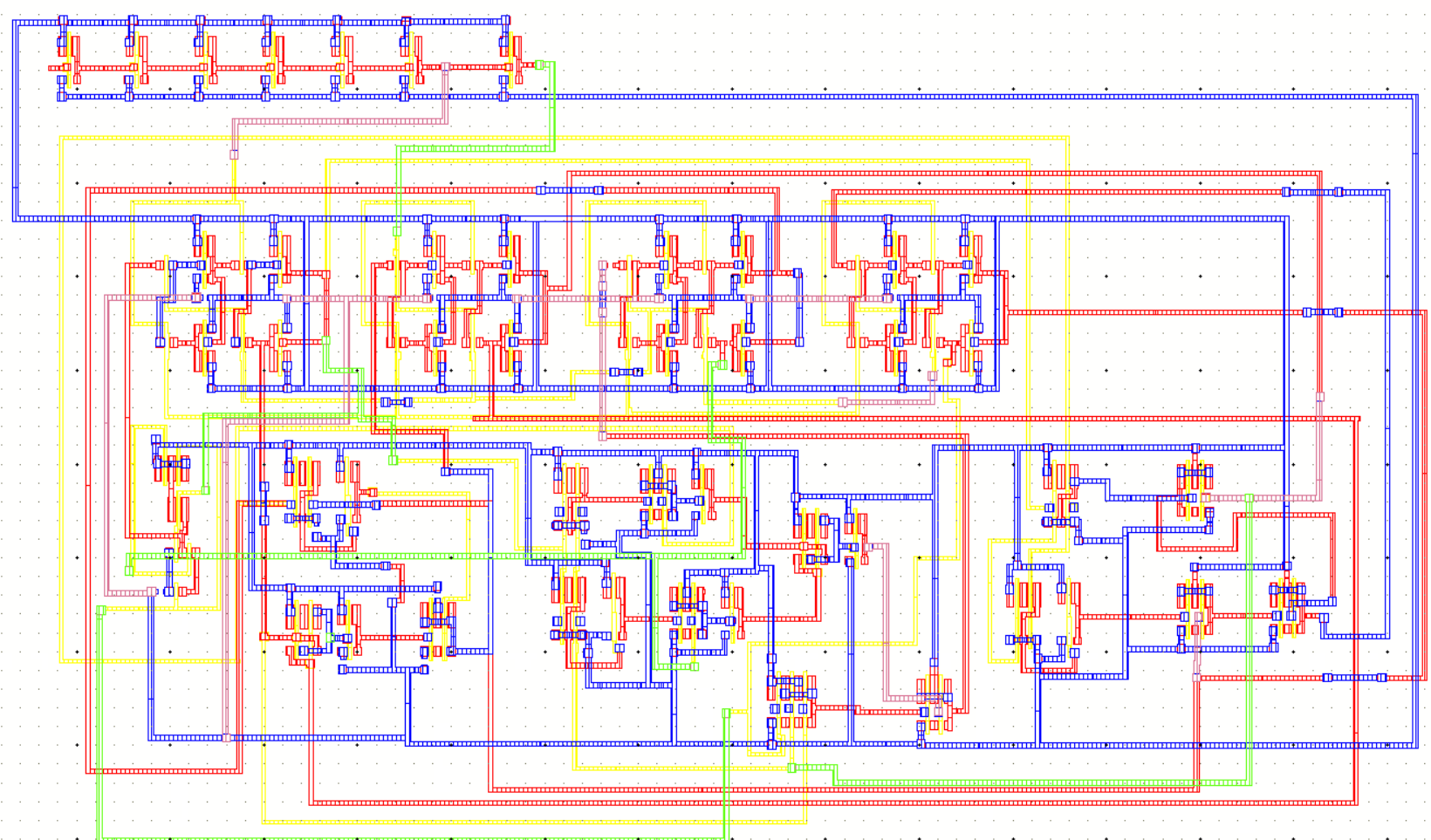

Fig 2.27 Av_extraction 


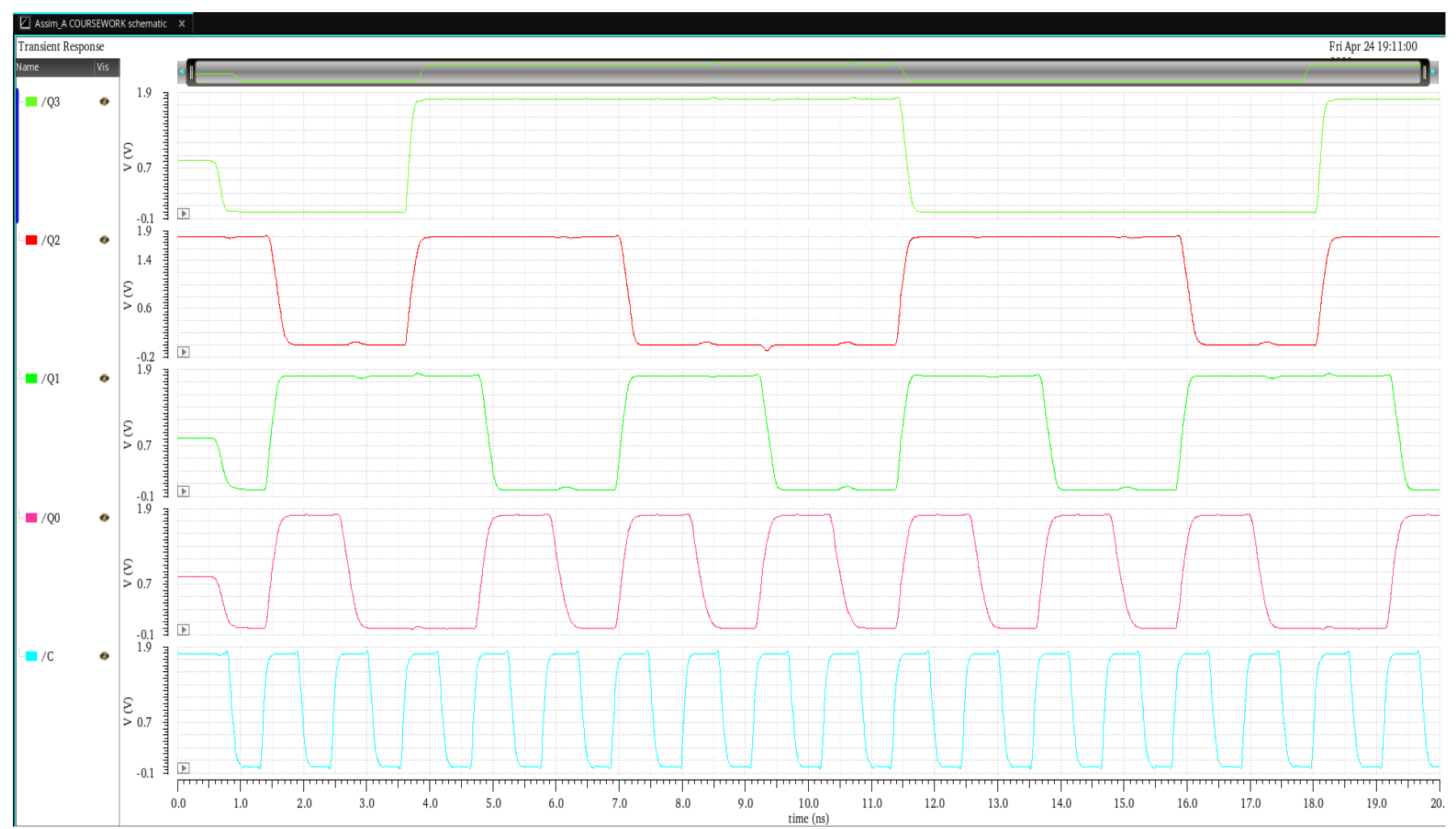

Fig 2.28 Simulation at layout level (av_extracted) at clock frequency $900 \mathrm{MHz}$

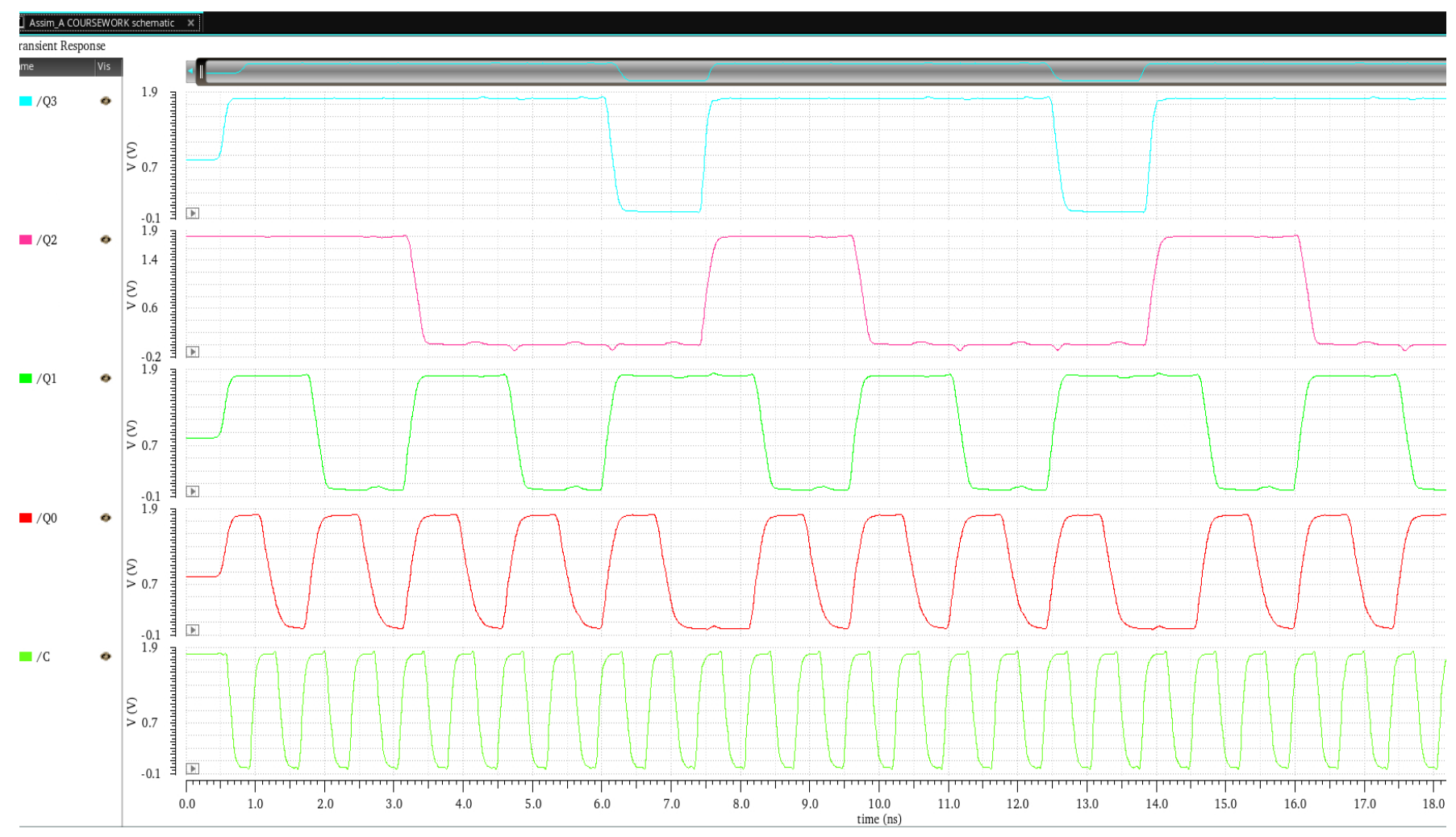

Fig 2.29 Simulation at layout level (av_extracted) at clock frequency $1.4 \mathrm{GHz}$ 


\section{Summary}

The transistor sizes used in the designs are provided below:

\begin{tabular}{|c|c|c|}
\hline & nMOS size & pMOS size \\
\hline Inverter & $180 \mathrm{~nm} / 240 \mathrm{~nm}$ & $180 \mathrm{~nm} / 1.13 \mathrm{um}$ \\
\hline Gates & $180 \mathrm{~nm} / 540 \mathrm{~nm}$ & $180 \mathrm{~nm} / 1.33 \mathrm{um}$ \\
\hline
\end{tabular}

The used VDD voltage for the entire system is $1.8 \mathrm{~V}$, this is the recommended value for this type of technology.

Number of used transistors per each element after optimizing expressions are as following:

\begin{tabular}{|c|c|}
\hline Element & Number of used transistors \\
\hline Clock generator (inverters) & 14 \\
\hline D trigger (per one) & $16 \times 4$ \\
\hline D0 output & 6 \\
\hline D1 output & 16 \\
\hline D2 output & 38 \\
\hline D3 output & 22 \\
\hline Total & $\mathbf{1 6 0}$ \\
\hline
\end{tabular}

\section{Conclusion}

The design of the MOD-13 down counter was successfully implemented using UMC_CMOS 180nm technology in Cadence Virtuoso, the counter starts counting at $14\left(1110_{2}\right)$ and ends at $2\left(0010_{2}\right)$. The maximum operating frequency is $1.4 \mathrm{GHz}$, using clock frequency higher than this value isn't allowed because the triggers start to work improperly, and the output signals will get distorted.

\section{Acknowledgement}

I would like to express my deep gratitude and appreciation to my professor, Dr. Morozov D. V. for his incredible support and patience throughout the semester, in Peter the Great Saint Petersburg Polytechnic University. Special thanks to my friends Nikolai, Timofey and Nosislav for their help. 


\section{References}

[1] Morozov D.V., Pilipko M.M., Design of Microelectronic Digital Circuits: tutorial - SaintPetersburg Polytechnic University Publishing House, 2014.

[2] S. S. Rais et al., "Characterization of Conventional Asynchronous Counters in Digital Systems," 2012 Fourth International Conference on Computational Intelligence, Modelling and Simulation, 2012, pp. 410-415, doi: 10.1109/CIMSim.2012.92.

[3] R. H. Seireg, A. E. Barbour and A. G. Vacroux, "A general approach to the design of modulo $\mathrm{N}$ asynchronous counters with 50\% duty cycle," Proceedings of the 32nd Midwest Symposium on Circuits and Systems, 1989, pp. 685-688 vol.2, doi: 10.1109/MWSCAS.1989.101947.

[4] A. Thakur and R. Mehra, "Power and speed efficient ripple counter design using $45 \mathrm{~nm}$ technology," 2016 IEEE 1st International Conference on Power Electronics, Intelligent Control and Energy Systems (ICPEICES), 2016, pp. 1-4, doi: 10.1109/ICPEICES.2016.7853183.

[5] R. Katreepalli and T. Haniotakis, "Energy-efficient synchronous counter design with minimum hardware overhead," 2017 International Conference on Communication and Signal Processing (ICCSP), 2017, pp. 1423-1427, doi: 10.1109/ICCSP.2017.8286619.

[6] Baker, R.J. (2010). CMOS circuit design, layout and simulation (3rd edition). Hoboken, NJ: John Wiley \& Sons, Inc.

[7] Sarkar, S., De, A. and Sarkar, S., 2014. Foundation Of Digital Electronics and Logic Design. Singapore: Pan Stanford Publishing, pp.141-170.

[8] Kumar, A., 2016. Fundamentals Of Digital Circuits - Delhi: Prentice-Hall of India. 